Annu. Rev. Mater. Res. 2016. 46:X--X doi: 10.1146/annurev-matsci-070115-031719

\title{
THERMAL BOUNDARY CONDUCTANCE: A Materials Science Perspective
}

\author{
Christian Monachon, ${ }^{1,2}$ Ludger Weber, ${ }^{3}$ and Chris Dames ${ }^{1,2, *}$ \\ ${ }^{1}$ Department of Mechanical Engineering, University of California, Berkeley, California \\ 94720; email: cdames@berkeley.edu \\ ${ }^{2}$ Materials Sciences Division, Lawrence Berkeley National Laboratory, Berkeley, California \\ 94720 \\ ${ }^{3}$ Laboratory of Mechanical Metallurgy, Ecole Polytechnique Fédérale de Lausanne, 1015 \\ Lausanne, Switzerland
}

\begin{abstract}
The thermal boundary conductance (TBC) of materials pairs in atomically intimate contact is reviewed as a practical guide for materials scientists. First, analytical and computational models of TBC are reviewed. Five measurement methods are then compared in terms of their sensitivity to TBC: the $3 \omega$ method, frequency- and time-domain thermoreflectance, the cut-bar method, and a composite effective thermal conductivity method. The heart of the review surveys 30 years of TBC measurements around room temperature, highlighting the materials science factors experimentally proven to influence TBC. These factors include the bulk dispersion relations, acoustic contrast, and interfacial chemistry and bonding. The measured TBCs are compared across a wide range of materials systems by using the maximum transmission limit, which with an attenuated transmission coefficient proves to be a good guideline for most clean, strongly bonded interfaces. Finally, opportunities for future research are discussed.
\end{abstract}


Keywords thermal boundary conductance, computational methods, experimental methods, interface materials science

\title{
INDEX OF VARIABLES
}

\author{
$h$ : thermal boundary conductance $(\mathrm{TBC})\left[\mathrm{W} /\left(\mathrm{m}^{2} \cdot \mathrm{K}\right)\right]$ \\ $L_{\mathrm{k}}$ : Kapitza length (m) \\ $p$ : polarization \\ $T$ : temperature $(\mathrm{K})$ \\ $\Delta T$ : temperature difference $(\mathrm{K})$ \\ $H$ : Heat carriers irradiance $\left(\mathrm{W} / \mathrm{m}^{2}\right)$ \\ $\omega$ : angular frequency $(\mathrm{rad} / \mathrm{s})$ \\ $\theta$ : incident angle (rad) \\ $\varphi$ : radial angle (rad) \\ $\eta$ : modewise carrier intensity $\left[\mathrm{W} /\left(\mathrm{m}^{2} \cdot \mathrm{sr}\right)\right]$ \\ $q$ : heat flux $\left(\mathrm{W} / \mathrm{m}^{2}\right)$ \\ $\alpha$ : carrier interfacial transmission coefficient \\ $f$ : Bose-Einstein occupation factor \\ $C$ : heat capacity per unit volume $\left(\mathrm{J} / \mathrm{m}^{3} \cdot \mathrm{K}\right)$ \\ $v$ : solid sound velocity $(\mathrm{m} / \mathrm{s})$ \\ $v$ : frequency $(\mathrm{Hz})$ \\ $\phi$ : lock-in amplifier phase angle \\ $X$ : lock-in amplifier in-phase signal \\ $Y$ : lock-in amplifier out-of-phase signal \\ $\kappa$ : thermal conductivity \\ $a$ : composite particle size \\ $m$ : measurable value \\ $S$ : sensitivity parameter
}

\section{SUBSCRIPT/SUPERSCRIPT}

int: interfacial

crit: critical

m: matrix

eff: effective 


\section{INTRODUCTION}

\subsection{Motivation and Applications}

Heat transfer through interfaces is becoming ever more important in modern technology. Indeed, although the relentless miniaturization of microelectronics and other functional devices enables their continued performance increases, it has also created a major problem in the thermal domain: Much effort is now needed to avoid overheating in the active area, which is crucial for semiconductor devices such as microprocessors, diodes, diode lasers, and highelectron-mobility transistors.

Miniaturization is thermally unfavorable for three reasons, as exemplified in microprocessors. (a) As the same functionality is achieved in a smaller device, the heat dissipation per unit volume increases. $(b)$ Functionality often actually increases with each technology generation. (c) The greater density of interfaces impedes the heat flow out of the device, which is further exacerbated by the shift to 3D architectures such as Intel's Tri-gate ${ }^{\circledR}$.

A critical scale for comparing bulk and interfacial thermal resistances is the Kapitza length, $L_{\mathrm{K}}$, defined as the ratio of the material's thermal conductivity $k$ to thermal boundary conductance (TBC). Using silicon as a representative benchmark for $k$, this crossover length for TBC dominance ranges widely, from as low as tens of nanometers for a high-quality $\mathrm{Pd} / \mathrm{Ir}$ interface (1) to as large as tens of micrometers for a highly mismatched Bi/diamond interface (2), values made even larger by any contamination at the interface ($)$. Because many modern devices rely on feature sizes and film thicknesses smaller than $L_{\mathrm{K}}$, the interfaces play an crucial role in limiting heat dissipation.

TBC is similarly important for many other modern semiconductor technologies that also contain a high density of nanoscale interfaces by design. Such technologies include optoelectronic devices such as quantum dot lasers (4), heat-assisted magnetic recording heads (5), and vertical cavity surface emitting lasers (6) and resonant diodes (7) based on superlattice Bragg mirrors. For these applications, thermal issues are critical because the maximum light output is limited by overheating of the active region. Similar issues arise in emerging fields such as organic electronics, which rely on metal contacts to cool the thermally insulating polymer device regions ( $\underline{8})$. TBC also dominates the heat transfer between 1D, 2D, and layered nanomaterials and their substrates (9--11), configurations being proposed for future electronic devices $(\underline{12}, \underline{13})$. TBC is also a limiting parameter in highelectron-mobility transistors, especially those on next-generation diamond substrates (14). 
TBC also plays an important yet underappreciated role in macroscopic thermal management materials. Hence, metal-matrix composites (MMCs) based on highly conductive inclusions (15) such as diamonds $(\underline{16}, \underline{17}), \mathrm{AlN}(\underline{18}), \mathrm{SiC}$, and carbon nanotubes (CNTs) (19) perform far below their full potential because their effective thermal conductivity is ultimately limited by the matrix-inclusion TBC (20). The low TBC at internal CNT-CNT junctions, as well as at their connections to the host polymer and surrounding boundaries, prevents the use of CNTs in thermal interface materials (21). Similarly, TBC is a crucial parameter in phase change materials for data storage (22) as well as in composites for thermal energy storage (23). TBC due to scattering at grain boundaries can also be a limiting factor for heat conduction in nanograined polycrystalline solids. On the other hand, low TBC can be exploited to tailor ultralow thermal conductivities by engineering nanolaminates (24) or nanocomposites (25), with potential applications including thermoelectric energy conversion and thermal barrier coatings.

\subsection{Scope and Related Work}

Several earlier reviews contain a wealth of information about TBC. The classic article by Swartz \& Pohl (26) summarized measurements and physical understanding of TBC at low and ultralow temperatures (0.1-30 K) developed through 1987 and presented the acoustic and diffuse mismatch models (AMM and DMM, respectively) and the phonon radiation limit $(\mathrm{RL})$, all of which are still in use today. Later, Cahill and coauthors $(\underline{27}, \underline{28})$ discussed TBC, including measurements, simulation, and selected experimental results, as part of broader reviews on nanoscale thermal transport. Finally, a recent review by Hopkins (29) summarized the effects of various experimental parameters on TBC, mainly that between metals and dielectrics.

The present article emphasizes the materials science of TBC and the phenomena around room temperature. We begin by briefly establishing the fundamental concepts and equations for the TBC between materials in intimate contact. TBC measurement techniques are then assessed in terms of their sensitivity. The article culminates by reviewing the TBC experimental literature from the past 30 years (see Figures 5-7 below), discussed from a materials science perspective. Throughout, the two essential themes for understanding TBC are the heat carrier irradiance and the interfacial chemistry and bonding. The thermal conductance of grain boundaries is not directly treated here (except briefly in section 3.3.2), though can be understood using many of the same principles as the ones discussed below. 


\section{THEORETICAL FOUNDATION: PHYSICAL ORIGINS OF TBC}

To introduce the theoretical concepts used to understand $\mathbf{T B C}$, we begin with a very general Landauer-Büttiker perspective. This approach highlights the two complementary factors determining the TBC in real experimental systems: the flux of energy carriers irradiating the interface and the ease of carrier transmission through the interface. We then discuss the strengths and limitations of the various approximations used to make the Landauer-Büttiker formulation tractable for simple calculations.

\subsection{Definition}

When a heat flux $J$ passes through a dense solid/solid interface, it causes a proportional temperature difference $\Delta T$ through it so that $J=h \Delta T$, where $h$ is the TBC between the two solids, expressed in $\mathrm{W} /\left(\mathrm{m}^{2} \cdot \mathrm{K}\right)$.

\subsection{Landauer-Büttiker Formulation: TBC Depends on Irradiance and Transmission Coefficient}

We present only the highlights of the main theory of TBC. More details are available in References $\underline{26}$ and $\underline{30}$. The equations are discussed in terms of phonons, but the fundamental concepts and results for electrons are identical after replacing Bose-Einstein statistics with Fermi-Dirac statistics. Similarly, we assume that both materials have isotropic dispersion relations, with a suitable generalization to highly anisotropic materials available in Reference $\underline{31 .}$

\subsubsection{Main Analytical Results.}

The essential picture is that of carrier bombardment and transmission (Figure 1b,c), which is readily analyzed using a Landauer-Büttiker formulation. This approach neglects coupled multicarrier effects and any inelastic scattering such as three-phonon processes at the interface. The interface $(x=0)$ is continually irradiated from both sides $(i=1,2)$ by phonons of various frequencies $(\omega)$, polarizations $(p)$, and directions $(\theta, \varphi)$. The total energy flux incident from side $i$ is the irradiance (in $\mathrm{W} / \mathrm{m}^{2}$ ),

$$
H_{i}=\sum_{p} \int_{\omega=0}^{\omega_{\max , i}} \int_{\varphi=0}^{2 \pi} \int_{\theta=0}^{\pi / 2} \eta_{i} f_{i} \sin \theta \mathrm{d} \theta \mathrm{d} \varphi \mathrm{d} \omega
$$


Here $f$ is the Bose-Einstein factor using temperature $T_{i}$, and the integration is over only the incident hemisphere. We also introduce a modewise intensity $\eta_{i}=\frac{1}{4 \pi} \mathrm{h} \omega v_{i} \cos \theta D_{i}$, where $\mathrm{h}$ is the reduced Planck's constant, $v_{i}(\omega, p)$ is the group velocity, and $D_{i}(\omega, p)$ is the density of states (DOS).

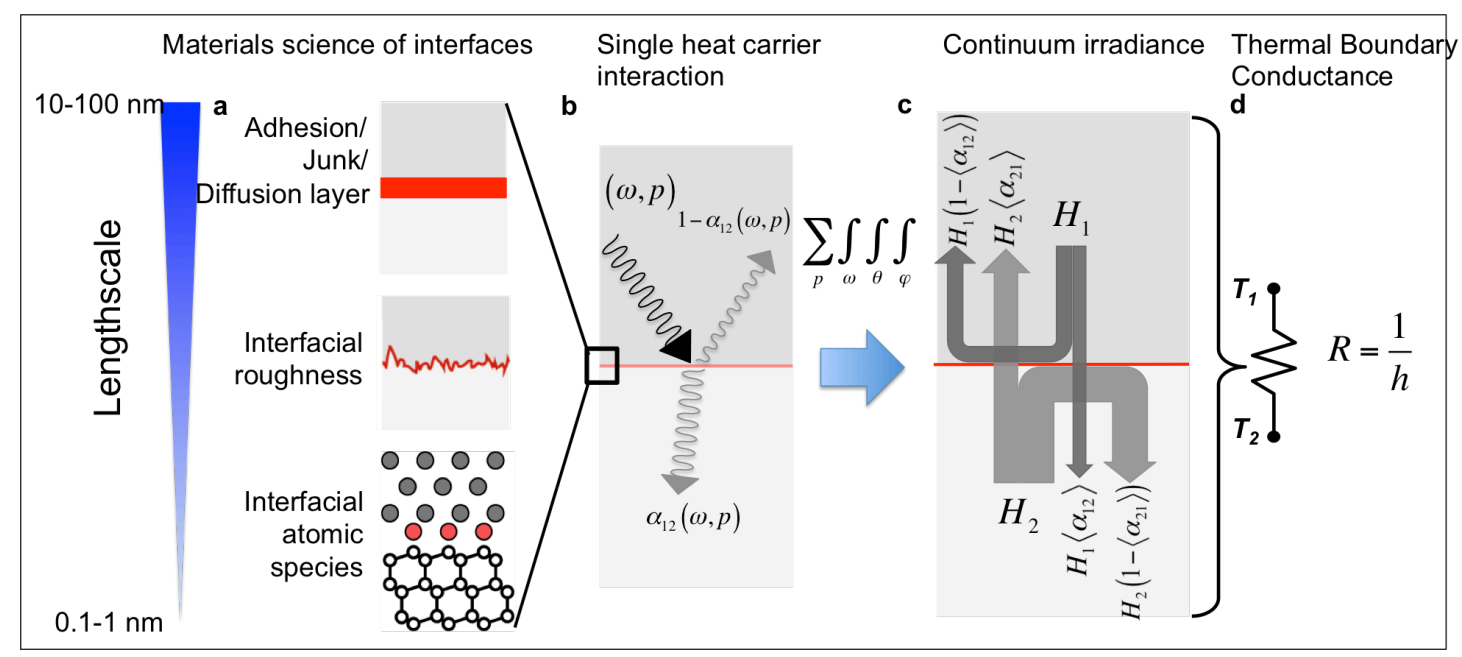

Figure 1 Relationship between microscopic and macroscopic pictures of thermal boundary conductance. $(a)$ The interface is defined by its micro- and nanostructural features. (b) An individual heat carrier of frequency $\omega$ and polarization $p$ strikes the interface, with a transmission probability $\alpha_{12}$. (c) Integrating over all carriers gives the total irradiance, $H_{1}$, with a weighted average transmission coefficient $\left\langle\alpha_{12}\right\rangle$. (d) The resulting heat transfer is equivalent to that of a simple thermal resistor.

Any given carrier (specified by $\omega, \theta, \phi, p$, and $i$ ) has a probability $\alpha_{i j}$ to transmit through the interface and deposit its energy in material $j$. If this $\alpha_{i j}$ is inserted into Equation 1 and the two sides are allowed to be at different temperatures, the net heat flux can be written as $q_{\text {net }}=q_{12}-q_{21}$, where

$$
q_{i j}=\sum_{p} \iiint_{\omega, \theta, \varphi} \eta_{i} f_{i} \alpha_{i j} \sin \theta \mathrm{d} \theta \mathrm{d} \varphi \mathrm{d} \omega
$$

with the angular integration again over the incident hemisphere. This microscopic formulation of $q_{\text {net }}$ is linked to the macroscopic irradiance picture of Figure $\mathbf{1 b}$ by defining an averaged transmission coefficient

$$
\alpha_{i j}=q_{i j} / H_{i},
$$

leading to

$$
q_{\text {net }}=\left[H_{1} \alpha_{12}\right]_{T_{1}}-\left[H_{2} \alpha_{21}\right]_{T_{2}},
$$

as illustrated in Figure 1b. At equilibrium, $q_{\text {net }}=0$, which requires $\left[H_{1} \alpha_{12}\right]_{T}=\left[H_{2} \alpha_{21}\right]_{T}(\underline{30})$. 
Finally, to obtain the linear response coefficient $h=\frac{\partial q_{\text {net }}}{\partial(\Delta T)}$, consider a small temperature difference across the interface and let $\Delta T \rightarrow 0$. Then

$$
h=\frac{\partial}{\partial T}\left[H_{1} \alpha_{12}\right]=\frac{\partial}{\partial T}\left[H_{2} \alpha_{21}\right]
$$

The equivalence of the $H_{1}$ and $H_{2}$ forms of Equation 5 means that TBC can be analyzed from the perspective of whichever material is more convenient. The other material still enters the problem, of course, through the transmission coefficients $\alpha_{i j}$.

Often $\eta_{i}$ and $\alpha_{i j}$ are independent of $T$, in which case

$$
h=\sum_{p} \iiint_{\omega, \theta, \varphi} \eta_{i} \frac{\partial f_{i}}{\partial T} \alpha_{i j} \sin \theta \mathrm{d} \theta \mathrm{d} \varphi \mathrm{d} \omega .
$$

This is a good approximation except near phase transition temperatures and in highly anharmonic materials.

Because the irradiation $H$ is likely unfamiliar to many readers (although it is a standard concept in radiation heat transfer), for physical insight it is helpful to consider a Debye model, which has a linear phonon dispersion of sound speed $v$. In this special case,

$$
\frac{\partial H_{i}}{\partial T}=\frac{1}{4} \sum_{p} C_{i, p} v_{i, p},
$$

where $C$ is the heat capacity per unit volume. Thus, we have the critical insight that TBC is closely related to a material's heat capacity and carrier velocities.

\subsubsection{THE PARADOX OF THE IMAGINARY INTERFACE: INCIDENT VERSUS EQUILIBRIUM}

TEMPERATURES. The main results for $h$ in Equations 5 and $\underline{6}$ have a conceptual flaw in the limit of an imaginary interface within a single material, which should give $h=\infty$. However, with $H_{1}=H_{2}$ and $\alpha=1$ for all carriers, Equations 5 and $\underline{6}$ clearly give a finite $h$. This is known as the Kapitza paradox. It can be resolved by redefining the local temperature to account for both incident and outgoing carriers (30). This approach is equivalent to multiplying the $h$ expressions from Equations 5 and $\underline{6}$ by a factor like $\left[1-\frac{1}{2}\left(\left\langle\alpha_{12}\right\rangle+\left\langle\alpha_{21}\right\rangle\right)\right]^{-1}(\underline{31})$. This temperature artifact correction can safely be ignored for most real interfaces. 


\subsection{The Three Quantities Controlling TBC}

$\underline{\text { Equations } 5}$ and $\underline{6}$ reveal the three main phenomena controlling TBC and are used throughout this review to interpret existing models and experiments. See also Figure 1.

2.3.1. DisPERSION RELATION. TBC scales with $H$, the irradiance of phonons bombarding the interface. This scaling calls for materials whose dispersion relations give large values of the $C \cdot v$ product (see Equation 7 ). At temperatures comparable to or higher than the Debye temperature $\Theta_{\mathrm{D}}$, the phonon $C$ approaches the constant DuLong-Petit limit. In this case, TBC tends to be higher in materials with stiffer bonding and lighter atoms because of the large sound velocities of such materials. Conversely, at very low temperatures TBC is maximized in materials with low sound velocities due to the $v^{3}$ dependence of the Debye heat capacity.

2.3.2. TEMPERATURE. Temperature has a direct impact through $\frac{\partial f}{\partial T}$ in Equation 6 or $C(T)$ in Equation 7. Thus, in phonon-dominated materials one finds $h \propto T^{3}$ for $T$ well below $\Theta_{\mathrm{D}}$, as thoroughly documented in Ref. 26 . For $T>\Theta_{\mathrm{D}}, h$ reaches a plateau in most cases $(49,53,128$, see also Figure 2), although some $T^{n}(0<n<1)$ behaviours have been reported in a few instances $(2,37)$.

2.3.3. INTERFACIAL BONDING. Finally, TBC is enhanced in materials pairs with strong interfacial coupling, which influences the transmission probabilities $\alpha_{i j}$. Although this is the most obvious mechanism influencing TBC, it is also by far the most challenging aspect to model accurately and control experimentally.

\subsection{TBC for Phonons: Analytical Expressions For Interface Transmission Coefficients}

Phonons are the main contributors to TBC at dielectric/dielectric and metal /dielectric interfaces (theoretical arguments about electronic contributions in the latter are briefly discussed in Section 2.5.2). We focus on phonons in crystalline solids. ${ }^{1}$

The large majority of analytical TBC formulae developed over the years are generalizations of three well-established approaches (26): the AMM, the DMM, and attempts to bound the TBC from above (namely the RL and the maximum transmission limit (MTL). The essential distinction between these models is the transmission coefficient $\alpha_{12}$ between the two materials.

\footnotetext{
${ }^{1}$ We are unaware of any model for the TBC in amorphous materials that considers non-propagating modes such as those introduced in Reference 164.
} 
2.4.1. Acoustic mismatch model. The AMM evaluates $\alpha_{12}$ by solving the continuum elasticity equations for the acoustic transmission and reflection between two linear elastic solids ( $\underline{32})$. Because this approach ignores the granularity of the lattice, it is most appropriate for $T \leq 30 \mathrm{~K}$ (26) so that long-wavelength phonons dominate the thermal spectrum. Each incident sound wave can be transmitted or reflected in six different ways (two longitudinal, four transverse) (33, pp. 311--43). Such complicated mode conversions are typically ignored, and the problem is simplified using expressions such as (르)

$$
\alpha_{12}(\theta, p)=\frac{4 \frac{\rho_{2} v_{p, 2}}{\rho_{1} v_{p, 1}} \frac{\cos \theta_{p, 2}}{\cos \theta_{p, 1}}}{\left(\frac{\rho_{2} v_{p, 2}}{\rho_{1} v_{p, 1}}+\frac{\cos \theta_{p, 2}}{\cos \theta_{p, 1}}\right)^{2}},
$$

where $\theta_{1}$ and $\theta_{2}$ are related through the acoustic analog of Snell's law, $\frac{\sin \theta_{1}}{v_{1}}=\frac{\sin \theta_{2}}{v_{2}}$, with the critical angle $\theta_{1, \text { crit }}=\sin ^{-1}\left(v_{1} / v_{2}\right)$ (assuming $\left.v_{2}>v_{1}\right)$.

Modifications of the AMM include a more complex treatment of sound waves ( $\underline{34})$ and taking into account weak interfacial bonding (35). Both of these modifications result in predicted $h$ values lower than those resulting from the traditional AMM, which in turn predicts values well below those from experimental measurements at room temperature $(\underline{2}, \underline{36})$.

2.4.2. Diffuse mismatch model. First proposed nearly 30 years ago, the DMM has been the most commonly used model to date $(\underline{2}, \underline{36}-\underline{39})$, albeit with mixed success. It postulates that all phonons impinging on the interface are scattered randomly, losing memory of their direction, polarization, and material of origin, keeping only their frequency $\omega$ constant. After such scattering, the probability for a phonon to propagate into material $i$ is proportional to that material's DOS at $\omega$. The DMM is thought more appropriate than the AMM at noncryogenic temperatures and for rough interfaces. This is because, at $300 \mathrm{~K}$ and up, the large majority of acoustic phonons have short wavelengths comparable to the interatomic spacing and surface roughness and can thus hardly see an interface as flat, as the AMM would require.

The DMM's postulate implies that the chances for a phonon incident from material 1 to transmit to material 2 are the same as those for a phonon from material 2 to be reflected at the interface. Thus, 


$$
\alpha_{i j}(\omega)=1-\alpha_{j i}(\omega)
$$

Combined with the assumed DOS weighting of the outgoing phonon rates, Equation 8 means that the DMM is specified purely by the two surrounding materials' bulk dispersion relations. $\alpha_{i j}(\omega)$ can then be found by applying Equation 8 to Equation 5 or $\underline{6}$. For example, for Debye solids at $T \ll \min \left(\theta_{\mathrm{D}, 1}, \theta_{\mathrm{D}, 2}\right)(\underline{26})$,

$$
\alpha_{12}=\frac{\sum_{p} v_{p, 2}^{-2}}{\sum_{p} v_{p, 1}^{-2}+\sum_{p} v_{p, 2}^{-2}}
$$

Among the many proposed extensions to the DMM include, most notably, the effect of interfacial reaction layers (40), the effect of interface roughening (41), and the effect of interfacial inelastic scattering (42). These treatments sometimes allow a good fit of specific results but, when exercised across a broader range of materials and interface types, have a predictive power that is limited at best.

2.4.3. Radiation and maximum transmission limits. The RL and the MTL were developed to assess upper limits to TBC. The RL (녀) assumes that all the phonons coming from the side with the lower DOS at a given frequency have a transmission of 1 . In the Debye approximation, this RL translates into

$$
h_{\mathrm{RL}}=\sum_{p, 2} \int_{\omega=0}^{\min \left(\omega_{\max }\right)} \int_{0}^{2 \pi} \int_{0}^{\pi / 2} \frac{\mathrm{h} \omega^{3}}{8 \pi^{3} v_{p, \max }^{2}} \frac{\partial f}{\partial T} \cos (\theta) \sin (\theta) \mathrm{d} \theta \mathrm{d} \phi \mathrm{d} \omega
$$

Note the sum runs over the three polarizations of material 2, whereas the frequency integration extends only up to the maximum frequency of material 1 . In the high-temperature limit, Equation 9 simplifies to $(\underline{2}, \underline{44})$

$$
h_{\mathrm{RL}}=\frac{k_{\mathrm{B}} \omega_{\max , 1}^{3}}{24 \pi^{2}} \sum_{p, 2}\left(\frac{1}{v_{p, \max }^{2}}\right) \text {. }
$$

The MTL (45) (equivalent to $G_{\max }$ in Reference 46 ) is very much in the same spirit but uses the irradiance $H$ (strictly speaking, its temperature derivative) and is generalized to allow perfect transmission of all phonons coming from the low- $\frac{\partial H}{\partial T}$ side (whether elastic or inelastic), hence setting the upper limit to $h$ for any conceivable interfacial scattering process subject only to the restrictions of the first and second laws of thermodynamics. Assuming that 
the materials are labeled such that $\frac{\partial H_{1}}{\partial T} \geq \frac{\partial H_{2}}{\partial T}$, we see from Equations 3 and $\underline{5}$ that the MTL therefore sets $\alpha_{21}=1$ to yield simply

$$
h_{\mathrm{MTL}}=\frac{\partial H_{2}}{\partial T} \text {. }
$$

For example, at $300 \mathrm{~K}, \mathrm{Cu}$ has $\frac{\partial H_{C u}}{\partial T} \cong 990 \mathrm{MW} /\left(\mathrm{m}^{2} \cdot \mathrm{K}\right)$ and diamond has $\frac{\partial H_{\text {Dia. }}}{\partial T} \cong 3000$ $\mathrm{MW} /\left(\mathrm{m}^{2} \cdot \mathrm{K}\right)$. Thus the corresponding MTL TBC is $h_{M T L, C u-D i a .}=990 \mathrm{MW} /\left(\mathrm{m}^{2} \cdot \mathrm{K}\right)$, limited by the phonon irradiance from the $\mathrm{Cu}$ side.

For a Debye model at high temperature, from Equation 7, this becomes:

$$
h_{\mathrm{MTL}, \text { Debye }}=\frac{1}{4} n_{2} k_{\mathrm{B}} \sum_{p} v_{s, i, 2}
$$

where $n_{2}$ is the atomic number density of the low- $H$ side and we use the DuLong-Petit heat capacity. The factor of $1 / 4$ in Equation 12 accounts for the angular integration of incident phonon velocities (26).

2.4.4. Influence of the solid's dispersion relation. The dispersion relations of the two materials clearly have a major influence on the calculated TBC. Because most models were originally developed using simple linear Debye models, some subsequent attempts have been made to incorporate more elaborate dispersion relations in models for $H_{i}$ and $\alpha_{i j}$. Most notably, the DMM has been revisited with various dispersion relations, including exact lattice dynamics (그), simplified lattice dynamics (ㅌ6), sine-type approximation (Born-von Karman) (45), truncated Debye (39), and fitted from measurements (48). Figure 2 shows the influence of the dispersion relation on the DMM for an $\mathrm{Al} / \mathrm{Si}$ system. 


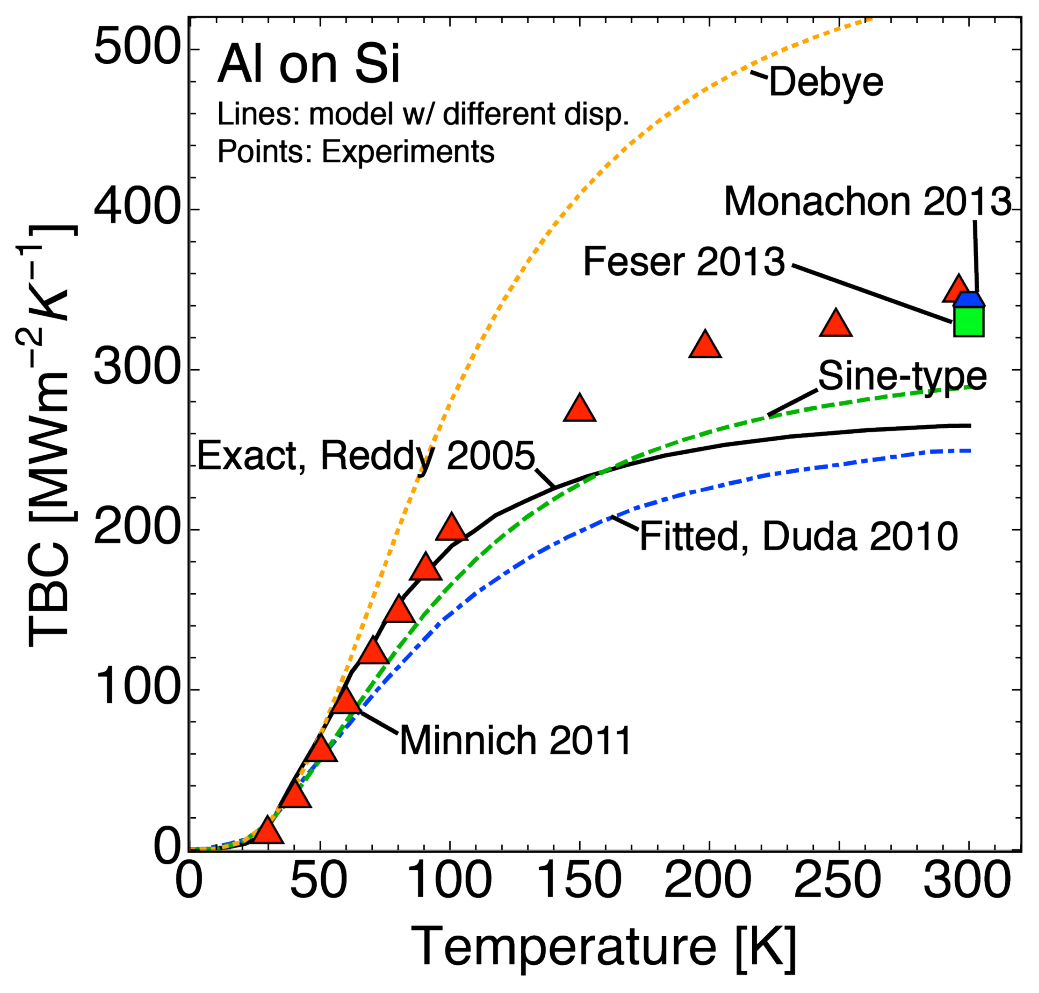

Figure 2 Effect of various dispersion relations on the diffuse mismatch model (DMM) for the TBC of a clean $\mathrm{Al} / \mathrm{Si}$ interface. The experimental values (red triangles) are taken from Minnich et al. (49), in excellent agreement with room-temperature measurements from Monachon and Weber (ref 128) (blue hexagon) and Wilson et al. (Ref 46) (green square). The DMM prediction using an exact phonon dispersion relation (black line) is taken from Reddy et al. (47). The prediction using a fitted $<100>$ dispersion relation (blue dashed-anddotted line) is taken from Duda et al. (48). The prediction using a sine-type dispersion relation (green dashed line) is computed according to a classic, Born-von Karman treatment of a 1D chain (45). In the Debye model (orange dashed line), the maximum frequencies for longitudinal and transverse branches were calculated for both materials by using $w_{\mathrm{D}}=v_{p}\left(6 \mathrm{p}^{2} n\right)^{1 / 3}$, with $n$ the atomic density, $p$ the polarization, and $v$ the sound velocity $(\underline{50)}$.

We note in passing that the DMM calculated using an exact dispersion relation fits the experimental data in Figure 2 very well up to $100 \mathrm{~K}$ and falls short by only approximately 20--30\% at higher temperatures. This is one of the most accurate TBC predictions using the DMM in the literature; many other DMM predictions fall only within a factor of 2 to 10 of the measured value.

\subsection{TBC for Electrons}

Electrons are responsible for most of the transport of heat in metals (무). Although the heat capacity of metals is very limited - a few percent of the total heat capacity of most metals at ambient $T$ - this limitation is outweighed by their high velocity. Most metals' Fermi velocities $\nu_{\mathrm{F}}$ are of the order of $10^{6} \mathrm{~m} / \mathrm{s}$, in contrast to phonon group velocities of the order of $10^{3} \mathrm{~m} / \mathrm{s}$ (50). Thus, in typical metals the $C \cdot v$ product is significantly higher for the electrons than the 
phonons, which in light of Equations 5, 7, and 11 explains why the TBC of metal-metal interfaces is expected to be dominated by the electrons.

2.5.1. Metal/Metal INTERFACES. The Landauer-Büttiker formalism presented in Equation 1 can be applied to an interface at which electrons dominate thermal transport, leading to the following electron irradiance $H_{\mathrm{e}}(\underline{51})$ :

$$
H_{\mathrm{e}}=\frac{1}{2} \int_{0}^{\pi / 2} \int_{0}^{\infty} E v_{\mathrm{F}, 1} N_{E, E_{\mathrm{F}, 1}, T} \alpha_{12}(E) \cos \theta \sin \theta \mathrm{d} \theta \mathrm{dE}
$$

Here $N_{E, E_{F, 1}, T}=\left(\exp \left(\left(E-E_{F, 1}\right) /\left(k_{B} T\right)\right)+1\right)^{-1}$ is the Fermi-Dirac function. From Expression 12, an MTL for electrons can be deduced as

$$
h_{\mathrm{MTL}}=\frac{1}{4} v_{\mathrm{F}} \gamma_{\mathrm{S}} T
$$

where $\gamma_{\mathrm{S}}$ is the material's Sommerfeld parameter (50). On the basis of this approach, Gundrum et al. (51) proposed an electronic version of the DMM that can be expressed as

$$
h_{\mathrm{el}}=\frac{\gamma_{\mathrm{S}, 1} v_{\mathrm{F}, 1} \gamma_{\mathrm{S}, 2} v_{\mathrm{F}, 2}}{4\left(\gamma_{\mathrm{S}, 1} v_{\mathrm{F}, 1}+\gamma_{\mathrm{S}, 2} v_{\mathrm{F}, 2}\right)} T
$$

This model gives accurate predictions in the case of $\mathrm{Al} / \mathrm{Cu}(\underline{51})$ and $\mathrm{Pd} / \mathrm{Ir}(\underline{1})$ metal/metal interfaces.

2.5.2. DieleCtric/Metal INTERFACES. Dielectric/metal interfaces impose two distinct challenges to an analysis of heat transfer. First, assuming that electrons do not contribute to TBC, a temperature difference can arise between the electrons and the phonons close to the surface (52). Although small, this effect may lead to errors in measuring dielectric/metal TBCs of the order of $500-1,000 \mathrm{MW} /\left(\mathrm{m}^{2} \cdot \mathrm{K}\right)(\underline{53})$. More broadly, this effect is observed in any case in which heat is transported through the interface by low-heat-capacity thermal excitations that are weakly coupled with the phonons on each side of the interface (54). Such excitations could include as electrons (e.g., metals), long-wavelength phonons (e.g., semiconductor alloys), or magnons.

Second, electrons on the metal side could conceivably pass some energy directly to phonons on the dielectric side of the interface. Proposed mechanisms include interaction with image charges in the dielectric (55). However, experiments by Stoner \& Maris (26) and Lyeo \& Cahill (2) show that a 400-fold change in electronic density for otherwise similar metals (Bi 
and $\mathrm{Pb}$ ) on the metal side of the interface does not impact TBC significantly, which is a strong argument against any such effect.

\subsection{Computational Approaches}

Computing thermal transport properties has gained substantial credibility in the past 20 years, thanks mainly to an increase in computing power and to the development of better simulation techniques (56). All computational techniques require three main inputs: a heat transportsolving algorithm, an interatomic potential, and a data extraction method. Each input impacts the final result, and their limitations should be known. Three main types of computational models have been used to describe TBC: $(a)$ molecular dynamics (MD) simulations, $(b)$ reciprocal space models, and (c) Green's function formalisms. Among these, at present only molecular dynamics can treat systems with interfacial structures close to reality; the other two need idealized settings such as perfectly epitaxial materials.

2.6.1. Interatomic potentials. Because many techniques use empirical interatomic potentials such as Lennard-Jones or Stillinger-Weber, any new user should bear in mind that these potentials are fit to reproduce only a subset of a material's properties, e.g., its bulk modulus and the size of its primitive unit cell. Calculating other properties is an extrapolation prone to potential artifacts. Density functional theory (DFT) and related methods $(\underline{57}, \underline{58})$ are a significant step forward but very costly computationally. Moreover, except if calculated using DFT, the nature of a bond can vary wildly at an interface, and the bonding to a different material can significantly influence the behavior of the few atomic layers surrounding the interface. Instructive literature examples can be found in Reference 59 for the diamond/metal interface and Reference $\underline{60}$ for an $\mathrm{Al} / \mathrm{Al}_{2} \mathrm{O}_{3}$ interface in which bonds that are a hybrid between metallic (on the $\mathrm{Al}$ side) and ionic (on the $\mathrm{Al}_{2} \mathrm{O}_{3}$ side) form in the last $\mathrm{Al}$ atomic layer. In both of these examples, an empirical potential would fail exactly where it needs to be most accurate: the interface. Thus, one should be extremely cautious when studying real systems beyond the idealized $\mathrm{Si} / \mathrm{Ge}$ interface.

2.6.2. Classical molecular Dynamics methods. MD methods use a system typically composed of several thousands of atoms with a given interatomic potential. Atomic motion is governed by simple equations of motion and follows Maxwell-Boltzmann classical statistics, limiting MD methods to $T>\theta_{\mathrm{D}} / 3(\underline{56})$. Temperature in MD is calculated by equating the local energy of the atoms to $k_{\mathrm{B}} T$, which requires large simulation cells (1ㅏ). Two main 
approaches, nonequilibrium molecular dynamics (NEMD) and equilibrium molecular dynamics (EMD), are used.

NEMD is typically characterized by hot and cold thermal reservoirs on either side of the simulation cell containing the interface of interest. Temperature is calculated over the length between the heat source and sink, and the interfacial temperature drop is extracted (62).

EMD can be used to determine thermal properties by looking at fluctuations of atomic energy around the equilibrium. TBC can be extracted using, e.g., the Green-Kubo formula (61).

Both MD techniques are relatively simple to implement and are useful to get insights into general trends. Both require large supercells to minimize size effects $(\underline{63})$ and a large number of time steps to ensure that the system is thermalized. NEMD techniques typically impose very high temperature gradients $\left(\sim 10^{9} \mathrm{~K} / \mathrm{m}\right)$, which may cause thermal transport to no longer be in a linear regime. EMD simulations inherently do not show that behavior but require longer simulation times $(\underline{61})$, which can significantly change results $(\underline{64})$.

2.6.3. RECIPROCAL SPACE MODELS. Reciprocal space models directly consider the phonons present in the two materials in contact. This approach has been used in two ways: $(a)$ lattice dynamics simulations $(\underline{36}, \underline{53}, \underline{65})$ and $(b)$ wave packet transmission calculations $(\underline{66}, \underline{67})$.

In lattice dynamics calculations, the two materials in contact are described by their structure and an interatomic potential, phonons of bulk dispersion are randomly generated in the each materials' first Brillouin zone, and continuity equations are applied at the interface. TBC can be deduced using the Landauer-Büttiker formalism presented in Equations 4 and $\underline{5}$.

Wave packet transmission calculations rely on a hybrid between a real-space approach and a reciprocal-space approach. These calculations consist of constructing an interface structure in real space, generating a wave packet at one end of it, and measuring the fraction of the wave packet that is transmitted through the interface (thereby bringing the analysis back into reciprocal space). This method provides a direct insight into the transmission coefficient but neglects phenomena related to phonon focusing (31) if not applied for a significant number of interfaces with various orientations or wave packet directions.

2.6.4. Atomistic Green's function. The Green's function formalism is a general method widely used in physics and applied mathematics to reduce complex problems involving differential equation to more tractable integral solutions. The goal of using Green's function is to calculate a harmonic matrix solution for the frequency and/or wave vector-dependent interface transmission properties. The full mathematical treatment for TBC and its advantages and limitations are reviewed in depth in Reference 68. Examples of application to TBC 
calculations in harmonic systems can be found in References $\underline{69}$ and $\underline{70}$. Anharmonic interactions can be taken into account only in simple systems ( $\underline{71})$. Inputs to this method are atomic masses and bonding strengths. A special class of these functions termed firstprinciples atomistic Green's functions use DFT-calculated force constants (72), permitting hope for quantitatively accurate results. In general, this treatment is well suited for ballistic transmission calculations, i.e., low-dimensional or low-temperature systems. Whereas the latter condition is not always fulfilled, the former may be for an interface, as in its simplest expression it consists of a spring connecting the two reservoirs. Thus, atomistic Green's functions can be used at higher temperatures if no inelastic interactions are assumed (68). The Green's function approach grows substantially in complexity as more degrees of freedom are added for the atoms, which means that low-dimensional (1D and 2D) systems are preferred (70).

\section{METHODS TO MEASURE TBC}

Here we survey the five main experimental methods used to measure TBC. Schematics of these methods are given at the top of Figure 3, and below in Figure 3 are maps of each method's sensitivity to TBC, discussed in detail below.

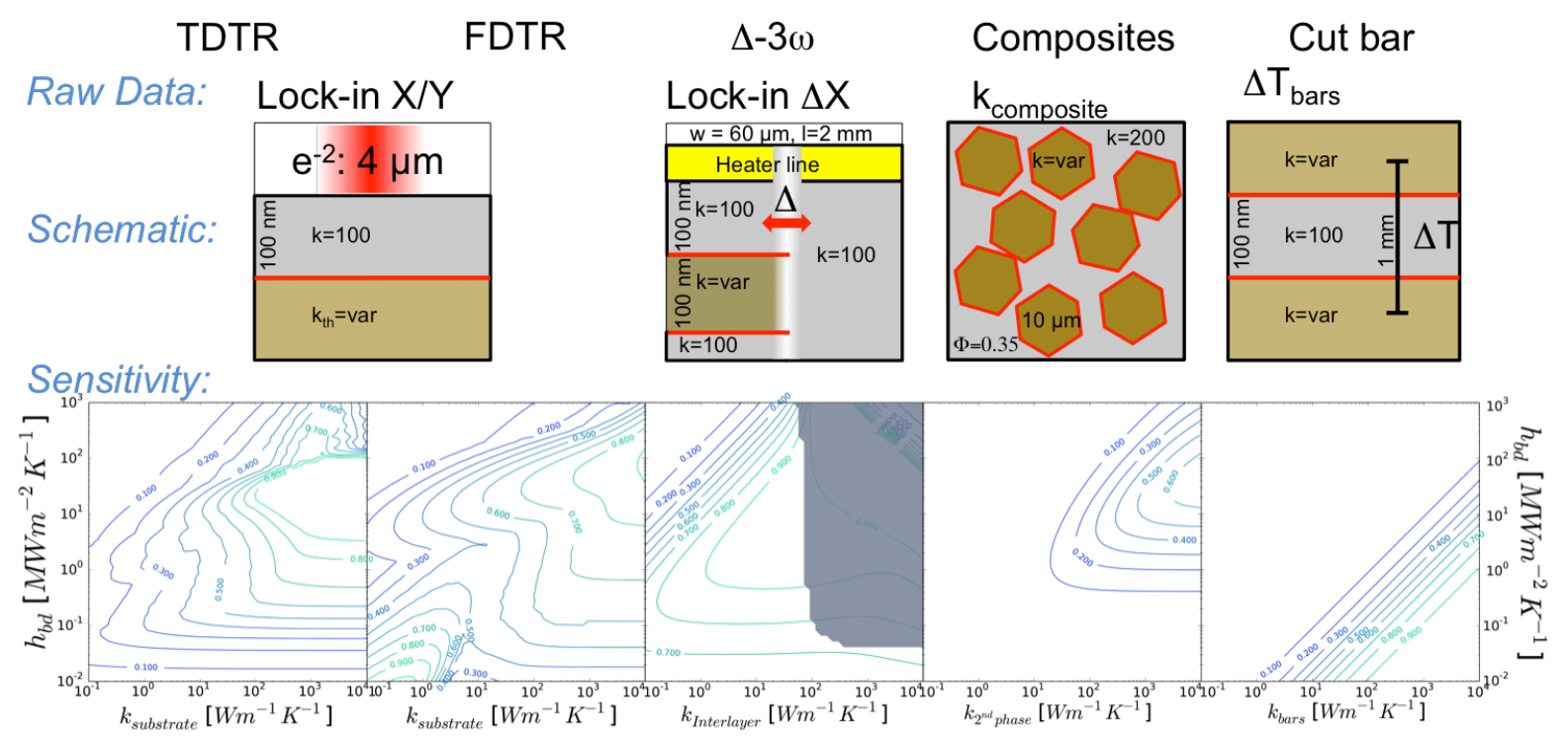

Figure 3 Overview of the five main experimental methods used to measure TBC, and their sensitivities. (First row) General name of the technique. Abbreviations: FDTR, frequency-domain thermoreflectance; TDTR, timedomain thermoreflectance. (Second row) The raw measurable, $m$, for which the sensitivity is evaluated. (Third row) Schematics, with the relevant TBC layers in red (the mentioned spot size is a radius). (Bottom row) Calculated sensitivity (contours of constant $S_{h}^{m}$; see Equation 20) of each technique's measurable to $h$. These maps are expressed as functions of two variables: $h$ and $k$ of the component indicated as $k=$ var. in each schematic. 


\subsection{Cut-Bar Method}

If the materials on either side of the interface are extremely conductive and the TBC very low, TBC can be measured with a simple cut-bar method. In practice, this high $k$ requirement restricts the method to cryogenic measurements, e.g., in the indium/sapphire system (그). At such temperatures, however, the mean free path of energy carriers can become comparable to the characteristic dimensions of the cut bars themselves, requiring careful thermocouple placement to avoid artifacts $(\underline{26})$.

\subsection{Thermally Modulated Methods}

Because TBC is restricted to an interface, methods capable of probing very small volumes are required. A widely exploited strategy is to use transient heating at short timescales, $t_{\text {char }}$, to control and minimize the thermal penetration depth

$$
d_{\text {th }} \sim \sqrt{\frac{k}{C} t_{\text {char }}},
$$

where $k$ is the thermal conductivity and $C$ the volumetric heat capacity. Localizing the thermal excitation and response to only the immediate vicinity of an interface makes the experiment more sensitive to TBC. This key insight is the basis of the three major TBC experimental schemes used today and described next: the electrothermal $3 \omega$ method and optical methods based on time- and frequency-domain thermoreflectance (TDTR and FDTR, respectively).

3.2.1. 3 $\omega$ AND RELATED ELECTROTHERMAL METHODS. This family of techniques uses a microfabricated metal strip to locally heat a dielectric substrate and uses the same or another nearby strip to measure the temperature response $(\underline{26}, \underline{74})$. The most prominent variant is the $3 \omega$ method $(\underline{74}, \underline{75})$, which uses an ac current at a frequency $\omega$ for heating, with the third harmonic voltage giving information about the thermal properties. Although applied primarily to measure $k$ of bulk substrates and thin films, in certain regimes the $3 \omega$ method is also sensitive to the TBC between the heater line and substrate (ㄷ--78).

In practice, the $3 \omega$ method is limited to frequencies $2 \pi \omega$ below $\sim 10-100 \mathrm{kHz}$, thereby placing a lower bound on $d_{\mathrm{th}}(\underline{14})$ and limiting the maximum TBC that can be detected. This method requires careful experimental design. A thin film is often incorporated between the substrate and heater line, so there are two relevant TBCs in series. The substrate should have high $k$. In one approach, the thermal response is measured as a function of the film thickness (whose $k$ must remain constant), allowing for a subsequent separation between interface and bulk 
contributions $(\underline{76}, \underline{79})$. If the intervening thin film is sufficiently thin and conductive $\left(d_{\text {film }} \cdot h\right.$ $\left.\ll k_{\text {film }}\right)$, the simpler differential $3 \omega$ method, which compares a pair of measurements with and without the film, may be used $(\underline{76}, \underline{79}, \underline{80})$.

3.2.2. Pump/Probe thermoreflectance Techniques. Pump/probe thermoreflectance techniques (thoroughly reviewed in Reference 1 1) are by far the most common for measuring TBC. Their core principle is to heat a 50--200-nm-thick metal layer on top of a substrate by using a modulated laser beam (the pump) while using another laser beam (the probe) to detect temperature through its effect on the metal's reflectance. Thermal models are then used to fit the obtained signal for one or more thermal properties of the system. The two main variants are FDTR and TDTR, and the results can be transformed between the two domains (82). In both variants, in contrast to the $3 \omega$ method, it is common practice to use both the in-phase $(X)$ and the out-of-phase $(Y)$ signal recorded by the lock-in amplifier, usually by plotting the phase angle $\phi=\tan ^{-1} \frac{Y}{X}$ or more directly the $\frac{X}{Y}$ ratio $(\underline{53}, \underline{81})$.

In FDTR ( $\underline{83}-\underline{85})$, both lasers are continuous wave $(\mathrm{CW})$, with the pump modulated at various frequencies. Compared to the ultrafast lasers used in TDTR, the CW lasers used in FDTR are cheaper and have greater wavelength flexibility but can be noisier. The optical alignment is simpler, except at very high frequencies (which can reach $200 \mathrm{MHz}$ ), which require matching the pump and probe path lengths to avoid residual phase errors.

In TDTR $(\underline{81}, \underline{86}, \underline{87})$, both lasers are pulsed, with pulse width of $0.1-10$ ps [using polymer dye lasers (36), Ti:sapphire lasers (2), or Nd:YAG ultrafast lasers (14)]. The pump is modulated at a single frequency to enable lock-in detection of the probe signal. Results are extracted by fitting curves of the probe signal as a function of the pump/probe delay time.

Alternative pump/probe techniques have also been used to investigate TBC. Siemens et al. (88) used a soft-X-ray probe's diffraction from nanofabricated Ni gratings to measure its TBC with $\mathrm{SiO}_{2}$ and sapphire. Highland et al. (9ㅜ) used X-rays to monitor the [004] diffraction peak of GaAs, to deduce its lattice temperature, and to obtain its $\mathrm{TBC}$ with neighboring $\mathrm{Al}$ layer. Finally, Hanisch-Blicharski et al. (0ㅡ) measured TBC at a Bi/Si interface by using the DebyeWaller factors of the Bi diffraction peaks.

\subsection{Effective Thermal Conductivity of Micro/Nanocomposites}

Another method to measure TBC comes from composites with high- $k_{i}$ inclusions in a low- $k_{\mathrm{m}}$ matrix (15). In these materials, a finite TBC reduces the overall $k$, an effect that can be used to 
measure it. Because this method has not been reviewed elsewhere, we give some details here. We begin with the theoretical basis of the TBC-extracting procedure, followed by examples of its application in the literature and a discussion of some of the associated difficulties. We conclude with a comparison of TBC values obtained using this inverse method as compared to direct methods such as TDTR.

3.3.1. TheOretical BaCKGROUND. Many models for calculating the effective composite thermal conductivity $k_{\text {comp }}$ have been developed over the last 150 years $(\underline{91}$, pp. 435--49; 92; 93) and are nicely summarized in References 94--97. Although most models were developed for random arrangements of spherical particles, they can be extended to spheroidal and ellipsoidal particles.

Although most models were originally developed for perfect conduction through the inclusion-matrix interfaces $(h=\infty)$, the effects of TBC can also be included (98--100). The key theoretical result is that, for spheres, adding a finite TBC to an inclusion with intrinsic $k_{i}^{\text {int }}$ leads to the same heat flow pattern as a spherical inclusion without TBC but using a modified effective conductivity, $k_{i}^{\text {eff }}$ :

$$
k_{i}^{\mathrm{eff}}=\frac{k_{i}^{\mathrm{int}}}{1+\frac{k_{i}^{\mathrm{int}}}{h a}},
$$

with $a$ the radius of the inclusion. Thus, $k_{\text {comp }}\left(k_{i}^{\text {int }}, h\right) \rightarrow k_{\text {comp }}\left(k_{i}^{\text {eff }}, h=\infty\right)$, allowing use of the numerous $k_{\text {comp }}$ models that assumed perfect interfaces. Equation 15 can also be used to extract the thermal conductance of grain boundaries for grains larger than mean free path of the material's heat carriers.

For spheroidal inclusions, the situation is less straightforward. However, for moderate contrast in $k$ (i.e., $k_{i}^{\text {eff }} / k_{m}<5$ ), and moderate spheroid aspect ratios (i.e., $<5$ ), for $k_{\text {comp }}$ calculations it is a reasonable approximation to replace randomly oriented spheroids with perfect spheres of the same hydraulic diameter, i.e., the same particle volume. We also note that, because the models described above are continuum models, they do not account for the reduction of $k_{i}^{\text {int }}$ in particles smaller than the intrinsic phonon mean free path [typically a concern only for $a$ smaller than $\sim 1-10 \mu \mathrm{m}(\underline{101})]$.

3.3.2. EXTRACTION PROCEDURES FOR TBC. The extraction of TBC from composite data is known as the inverse problem (102). Obtaining TBC from composite thermal conductivities 
can be achieved either through an implicit procedure, i.e., directly fitting a set of composite $k$ for TBC (102--105), or through explicit extraction of the effective thermal conductivity of the inclusion (106--109). Rearranging Equation 15 gives

$$
\frac{1}{k_{i}^{\mathrm{eff}}}=\frac{1}{k_{i}^{\mathrm{int}}}+\frac{1}{h a}
$$

Hence, plotting $\left(k_{i}^{\text {eff }}\right)^{-1}$ against $a^{1}$ should yield a line of slope $h^{1}$ and an intercept at $a^{1}=$ 0 of $\left(k_{i}^{\text {int }}\right)^{-1}$. This procedure has been successfully applied in, e.g., $\mathrm{M}(\mathrm{M}=\mathrm{Al}, \mathrm{Cu}$, $\mathrm{Sn}) / \mathrm{AlN}$ systems $(\underline{18})$ and $\mathrm{M}(\mathrm{M}=\mathrm{Al}, \mathrm{Al}-\mathrm{Si}, \mathrm{Ag}, \mathrm{Ag}-\mathrm{Si}) /$ diamond systems $(\underline{106}, \underline{107})$, with representative data in Figure 4.
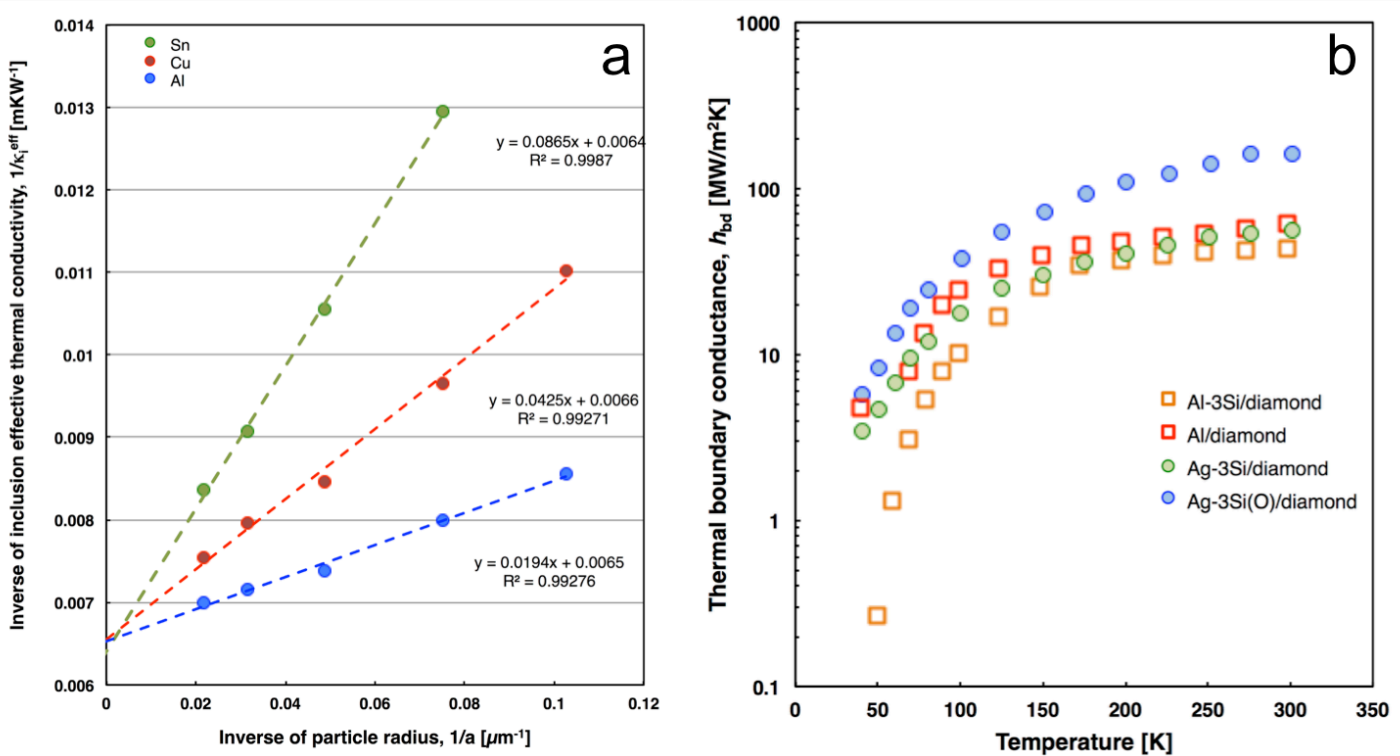

Figure $4(a)$ Graphical extraction of TBC by plotting the effective thermal conductivity of AlN inclusions, extracted from $k_{\text {comp }}$ using a differential effective medium scheme, as a function of the inverse of the equivalent particle radius. The slope of the linear fit is $1 / h$, and the intercept gives $\left(k_{i}^{\text {int }}\right)^{-1}$. Experimental data for AlN particles in various matrix metals from Kida et al. (18). (b) TBC extracted from composites with different matrix alloys and four different particle sizes by using Equation 15. The characteristic decrease in TBC below $300 \mathrm{~K}$ is consistent with theory and other techniques of TBC measurement (e.g., Figure 2). Data compiled from Edtmaier et al. $(\underline{106}, \underline{107})$.

A related approach $(110,111)$ determines the critical inclusion size, $a_{\text {crit, }}$ at which $k_{\text {comp }}=k_{\mathrm{m}}$ (112). Setting $k_{\mathrm{m}}=k_{\mathrm{i}}^{\text {eff }}$ and $a=a_{\text {crit }}$ in Equation 15 leads to

$$
h=\frac{1}{a_{\text {crit }}} \frac{k_{i}^{\mathrm{int}} k_{\mathrm{m}}}{k_{i}^{\mathrm{int}}-k_{\mathrm{m}}}
$$

Given measurements of $k_{\text {comp }}$ as a function of $a$ for a fixed particle volume fraction, the point at which $k_{\text {comp }}=k_{\mathrm{m}}$ determines $a_{\text {crit, }}$ and thus $h$, from Equation 17. 


\subsubsection{Challenges IN eXtraCting TBC FROM COMPOSITE Data. It is becoming} customary in the composite literature to report TBC of matrix/inclusion couples extracted from $k_{\text {comp }}$, sometimes even from single experiments. The uncertainties with such procedure are, however, significant:

- A 1-3\% uncertainty lies in the measurement of $k_{\text {comp, }}$, which can easily translate into a $3-5 \%$ uncertainty in $k_{i}^{\text {eff }}$.

- Uncertainty in $k_{i}^{\text {eff }}$ is introduced by the uncertainty in composite parameters, e.g., matrix thermal conductivity, inclusion volume fraction, and the presence of pores. This uncertainty can be alleviated by concomitant density and matrix electrical conductivity measurements (see, e.g., References $\underline{109}$ and $\underline{113}$ ), but samples containing more than 1-vol\% porosity should be avoided altogether.

- Different modeling schemes give differing results for $k_{\text {comp }}$. The often-used formula proposed by Hasselmann \& Johnson (114), an explicit form of the Maxwell-Garnett mean field scheme, overestimates the effective thermal conductivity for insulating inclusions $(\underline{20}, \underline{115})$. In cases of large phase contrast, $k_{i}^{\text {eff }} / k_{m}>4$, the differential effective medium scheme should be used instead (20).

Once the effective $k_{i}^{\text {eff }}$ is obtained, the effects of the uncertainty in the intervening parameters on the uncertainty in the extracted $h$ can be assessed. Rearranging Equation 1 to isolate $h$ and taking the total differential as the uncertainty on TBC, $\Delta h$, we find

$$
\Delta h=\left|\frac{k_{i}^{\text {int }} k_{i}^{\text {eff }}}{\left(k_{i}^{\text {int }}-k_{i}^{\text {eff }}\right)} \frac{1}{a^{2}}\right| \Delta a+\left|\frac{\left(k_{i}^{\text {eff }}\right)^{2}}{\left(k_{i}^{\text {int }}-k_{i}^{\text {eff }}\right)^{2}} \frac{1}{a}\right| \Delta k_{i}^{\text {int }}+\left|\frac{\left(k_{i}^{\text {int }}\right)^{2}}{\left(k_{i}^{\text {int }}-k_{i}^{\text {eff }}\right)^{2}} \frac{1}{a}\right| \Delta k_{i}^{\text {eff }}
$$

Again, upon rearranging Equation 15 to eliminate the $\left(k_{i}^{\text {int }}-k_{i}^{\text {eff }}\right)$ terms and inserting the result into Equation 18, we find the relative error on $\mathrm{TBC}$ to be

$$
\frac{\Delta h}{h}=\left|\frac{\Delta a}{a}\right|+B i\left|\frac{\Delta k_{i}^{\text {int }}}{k_{i}^{\text {int }}}\right|+\frac{B i^{2}}{B i+1}\left|\frac{\Delta k_{i}^{\text {eff }}}{k_{i}^{\text {eff }}}\right|
$$

with $B i$ the Biot number of the inclusion given by $h a / k_{i}^{\text {int }}$.

Hence, for $B i<1$, i.e., for strong influence of interfaces, the main source of error in $h$ comes from the relative uncertainty in inclusion size $a(\underline{116})$. For $B i>10$, uncertainties in the intrinsic properties of the inclusions $k_{i}^{\text {int }}$ are dominant. This is particularly important for industrially produced diamond, $\mathrm{cBN}$, and $\mathrm{SiC}$, as deviation from stoichiometry (cBN, $\mathrm{SiC}$ ) 
and impurities (mainly nitrogen in synthetic diamond) lead to significant uncertainty in $k_{i}^{\text {int }}$ $(\underline{117}, \underline{118})$.

3.3.4. COMPARISON OF DIRECT AND INDIRECT DETERMINATION OF TBC - The indirect method of extracting TBC has been applied mainly to metal/dielectric combinations motivated by thermal management applications, e.g., Al/SiC (102, 108--110, 113), (Cu, Ag, $\mathrm{Al}, \mathrm{ZnS}$, cordierite)/diamond (16, 20, $\underline{106}, \underline{110}, \underline{119}-\underline{-121})$, and (Cu, $\mathrm{Al}) / \mathrm{AlN}(\underline{18})$. Virtually all the cited references give TBC around room temperature; the exceptions are Hasselmann and colleagues $(113,122)$ for the diamond/cordierite and $\mathrm{Al} / \mathrm{SiC}$ systems and Edtmaier et al. $(\underline{106}, \underline{107})$ for several metal/diamond systems shown in Figure 4.

Table 1 compares the composite TBC extraction scheme and TDTR on equivalent systems. A more complete table of TBC values can be found in Supplemental Table $2 . h_{\text {comp }}$ is within 20 $-30 \%$ of $h_{\mathrm{TDTR}}$ in every case, which is noteworthy considering that the composites' synthesis route involves very high temperatures, which can significantly change the interface's nature compared with interfaces created in thin-film deposition.

Table 1 Comparisons of TBC determined from direct (TDTR) and indirect (composite) methods, for couples of $\mathrm{Al}$ on $\mathrm{SiC}, \mathrm{AlN}$, and hydrogen-terminated diamond

\begin{tabular}{|l|l|l|}
\hline Couple & $\boldsymbol{h}_{\text {comp }}\left[\mathbf{M W} /\left(\mathbf{m}^{\mathbf{2}} \cdot \mathbf{K}\right)\right]$ & $\boldsymbol{h}_{\text {TDTR }}\left[\mathbf{M W} /\left(\mathbf{m}^{\mathbf{2}} \cdot \mathbf{K}\right)\right]$ \\
\hline $\mathrm{A} 1 / \mathrm{SiC}$ & $\begin{array}{c}146(\underline{123}), 140(\underline{109}), 220(\underline{113}), 180 \\
(\underline{102})\end{array}$ & $200(\underline{39})$ \\
& & $47(\underline{124})$ \\
\hline $\mathrm{A} 1 / \mathrm{AlN}$ & $50(\underline{18})$ & $\begin{array}{l}32--65(\underline{125}), 22 \\
(126)\end{array}$ \\
\hline $\mathrm{Al} / \mathrm{C}: \mathrm{H}$ & $62(\underline{106}), 5--45(\underline{121})$ & \\
\hline
\end{tabular}

\subsection{Comparison of the Sensitivities of the TBC Measurement Methods}

To summarize the relative strengths and practical operating regimes of the five major experimental methods presented above, Figure 3 presents calculations of each method's sensitivity to $\mathbf{T B C}$, using realistic properties and geometries. The sensitivity $S$ of measurable $m$ to parameter $p$ is

$$
S_{p}^{m}=\frac{\mathrm{d} \ln (m)}{\mathrm{d} \ln (p)} \quad S_{p}^{m}=\frac{\partial \ln (m)}{\partial \ln (p)}=\frac{p}{m} \frac{\partial m}{\partial p},
$$

where here we focus on $p=h$ and the relevant $m$ for each method is specified in Figure 3. This comparison gives helpful insights into which method to use in which case. For a more 
complete understanding, the selectivity, i.e., the ability of the technique to discriminate between e.g., $k_{\text {sub }}$ and $h$, should also be considered. Moreover, the uncertainty in $k_{\text {sub }}$ can propagate into errors in $h$ through $\left(\frac{\Delta h}{h}\right)=\left(\frac{S_{k_{\text {sub }}}^{m}}{S_{h}^{m}}\right)\left(\frac{\Delta k_{\text {sub }}}{k_{\text {sub }}}\right)$ (127). Some specific observations from Figure 3 follow.

- TDTR and FDTR are the most versatile methods, with TDTR being more sensitive for a wide range of intermediate $h$. By having access to both time and frequency information, TDTR has in general a better selectivity to $h$ than does FDTR (details not shown in Figure 3). As a side note, TDTR also has the benefit of measuring thermal transport as fast as sub-ns scales, corresponding to $\mathrm{GHz}$ frequency bandwidth, as compared to $200 \mathrm{MHz}$ and less for FDTR.

- The differential $3 \omega$ method is appropriate for measurements of moderate to low $h$ and high $k_{\text {sub }}$. For some $k_{\text {interlayer }} / h$ combinations, the effective thermal conductivity of the variable- $k$ film (including its TBCs) matches that of the substrate material. In this special case $\left|S_{h}^{\operatorname{Lock}-\operatorname{In} \Delta X}\right| \rightarrow \infty$, but this regime is impractical because the $3 \omega$ voltage difference $\Delta X$ vanishes and is thus overwhelmed by noise. To exclude this artifact, a shaded area was overlaid on Figure 3 where $\Delta X$ is expected to be less than $1 \mu \mathrm{V}$.

- The composite approach [using the recommended (20) differential effective medium model] is sensitive to TBC when the inclusions conduct heat at least as well as the matrix ( $\Phi k_{i}^{\text {eff }} \geq k_{m}$, where $\Phi$ is the volume fraction of particles), and requires TBC to not screen the inclusions, resulting in $h \sim k_{i}^{\text {int }} / a$ for maximum sensitivity.

- The cut-bar method is valid only for high $k_{\text {bars }}$ and low $h$, consistent with cryogenic temperature measurements (26).

\section{MAIN FACTORS EXPERIMENTALLY PROVEN TO INFLUENCE TBC}

We conclude this review by summarizing high-quality TBC measurements from the literature, interpreted using the framework established in Section 2. With reference to Figure 1, the two essential materials science considerations are the carrier irradiances, $H_{i}$, and the interface transmissivities, $\left\langle\alpha_{i j}\right\rangle$. Sections 4.2 and 4.5 focus on the effects of phonon dispersion relation 
on $H$, whereas Sections 4.3-4.8 deal with the effects of interfacial bonding and quality on $\langle\alpha\rangle$.

\subsection{Benchmarking}

The TBC between $\mathrm{Al}$ and clean sapphire has been measured by a number of investigators $(\underline{36}$, $\underline{37}, \underline{128})$, with good convergence around $h=185 \pm 15 \mathrm{MW} /\left(\mathrm{m}^{2} \cdot \mathrm{K}\right)$ at $300 \mathrm{~K}$. This system is particularly suitable for benchmarking because sapphire is commercially available with very low surface roughness, is easily cleaned by baking in air at high $T$, and does not react with Al. The Al/Si couple has also been widely studied. Although the interfacial quality is harder to control, measurements for the highest-quality interfaces cluster around $h=345 \pm 40$ $\mathrm{MW} /\left(\mathrm{m}^{2} \cdot \mathrm{K}\right)$ at $300 \mathrm{~K}($ Figure 2) (⒐ $)$.

\subsection{Dispersion Relation and Acoustic Contrast}

One important trend of the experimental literature is that high dissimilarity between the phonon properties of two materials is consistently found to yield low TBC. Such results have traditionally been presented and interpreted in terms of the contrast between the materials' Debye temperatures or, equivalently, their maximum phonon frequencies, such as the results collected in Figure 5 for various metals on diamond. The $\theta_{\mathrm{D}}$ ratios can be large, exceeding 10:1 in couples pairing dense, soft metals like $\mathrm{Bi}, \mathrm{Pb}$, and $\mathrm{Au}\left(\theta_{\mathrm{D}}=95,102\right.$, and $165 \mathrm{~K}$, respectively) with diamond $\left(\theta_{\mathrm{D}}=2,240 \mathrm{~K}\right)(\underline{2}, \underline{36})$ or graphite $(\underline{134}, \underline{135})$. The $\mathbf{R L}$ picture holds for most systems at cryogenic temperatures [lower than approximately $\left(\theta_{\mathrm{D}, \min } / 10\right]$, where the AMM shows excellent results (see Reference 26 and references therein). However, at room temperature, as shown in Figure 5 the correlation between $\theta_{\mathrm{D} \text {,min }}$ (indicated by the $\omega_{\max }$ axis) and TBC is modest at best, with the interfacial quality having a larger influence in most cases, as discussed further in Section 4.3. Figure 5 also shows that the MTL is better than the RL as an upper bound and for describing the observed experimental trends, suggesting that inelastic interfacial scattering processes are important at room temperature and above. 


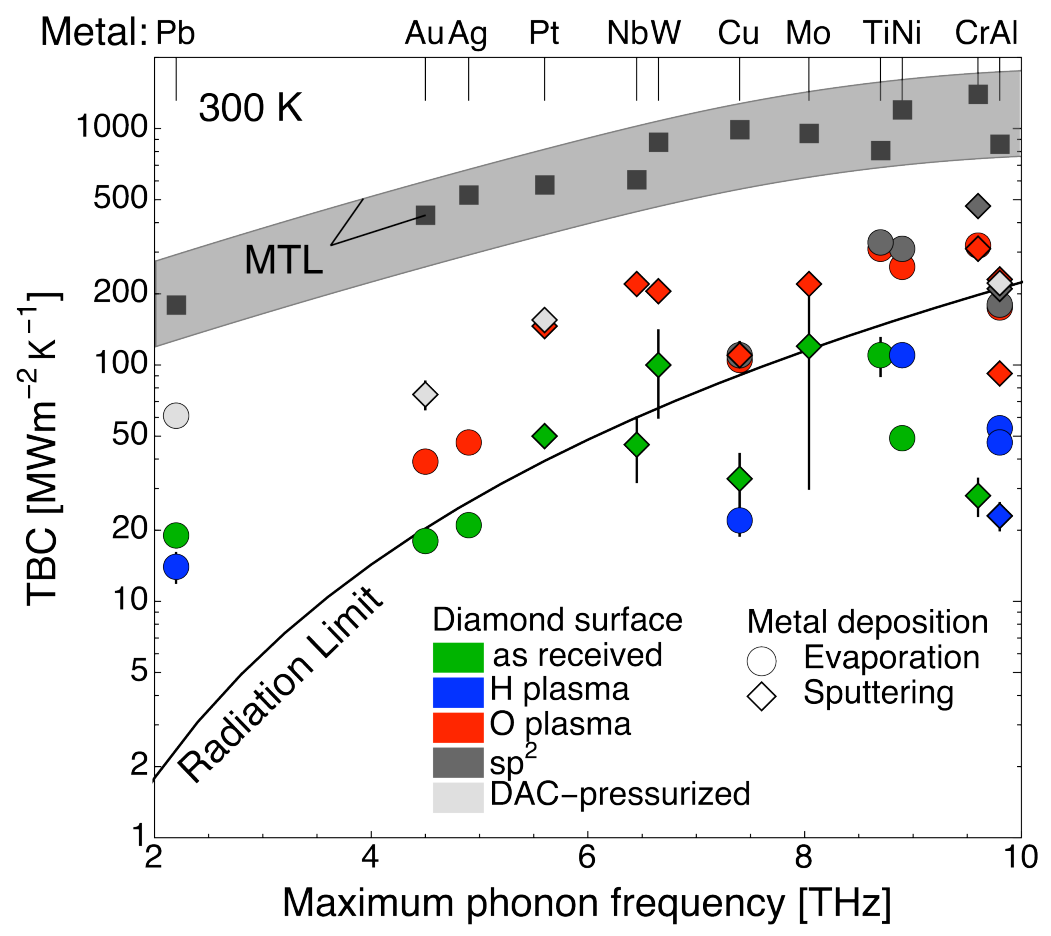

Figure 5 Collected experimental results for the TBC between diamond and 12 different metals as a function of the maximum phonon frequency of the metal (collected from http://materials.springer.com/) along the bottom axis, with the specific metals indicated by the ticks along the top axis. Point color and shape indicate surface treatment and metal deposition technique, respectively. For modeling context, the solid line is the radiation limit (Equation 10), and the black squares and shaded band show the maximum transmission limit (MTL) (Equation 11). The available error bars indicate standard deviation over four measurements. Diamond anvil cell (DAC)pressurized values from Reference $130, \mathrm{~Pb}$ /diamond values from Reference 2 , and the lowest $\mathrm{H}$ - and $\mathrm{O}$ - plasma $\mathrm{Al} /$ diamond values from Reference $\underline{126}$. Other values from References $\underline{44}, \underline{125}, \underline{128}$, and 131--133.

Figure 6 is the most comprehensive of this review, summarizing TBC measurements for 34 distinct couples with high-quality interfaces; these measurements were collected from 19 primary references. For both phonons and electrons, the dominant trend is that the measured $h$ correlates with the irradiance temperature derivative $(\underline{45}, \underline{46})$ from the weaker side, consistent with the physical picture established in Figure 1. Using $\left[\frac{\partial H}{\partial T}\right]_{\min }$ as the $x$-axis is advantageous because $\left[\frac{\partial H}{\partial T}\right]_{\min }$ depends only on the dispersion relation of a single material and makes no assumptions about the detailed transmission physics of $\alpha$. The 1:1 line is the MTL, corresponding to $\alpha_{\min \rightarrow \max }=1$ and $h=\left[\frac{\partial H}{\partial T}\right]_{\min }$. Figure 6 shows that the large majority of available experiments on high-quality interfaces can be understood in terms of the minimum irradiance picture; the corresponding average transmission coefficients $\left\langle\alpha_{\min \rightarrow \max }\right\rangle$ are in the range $0.1-0.5$, the details of which are discussed next. 


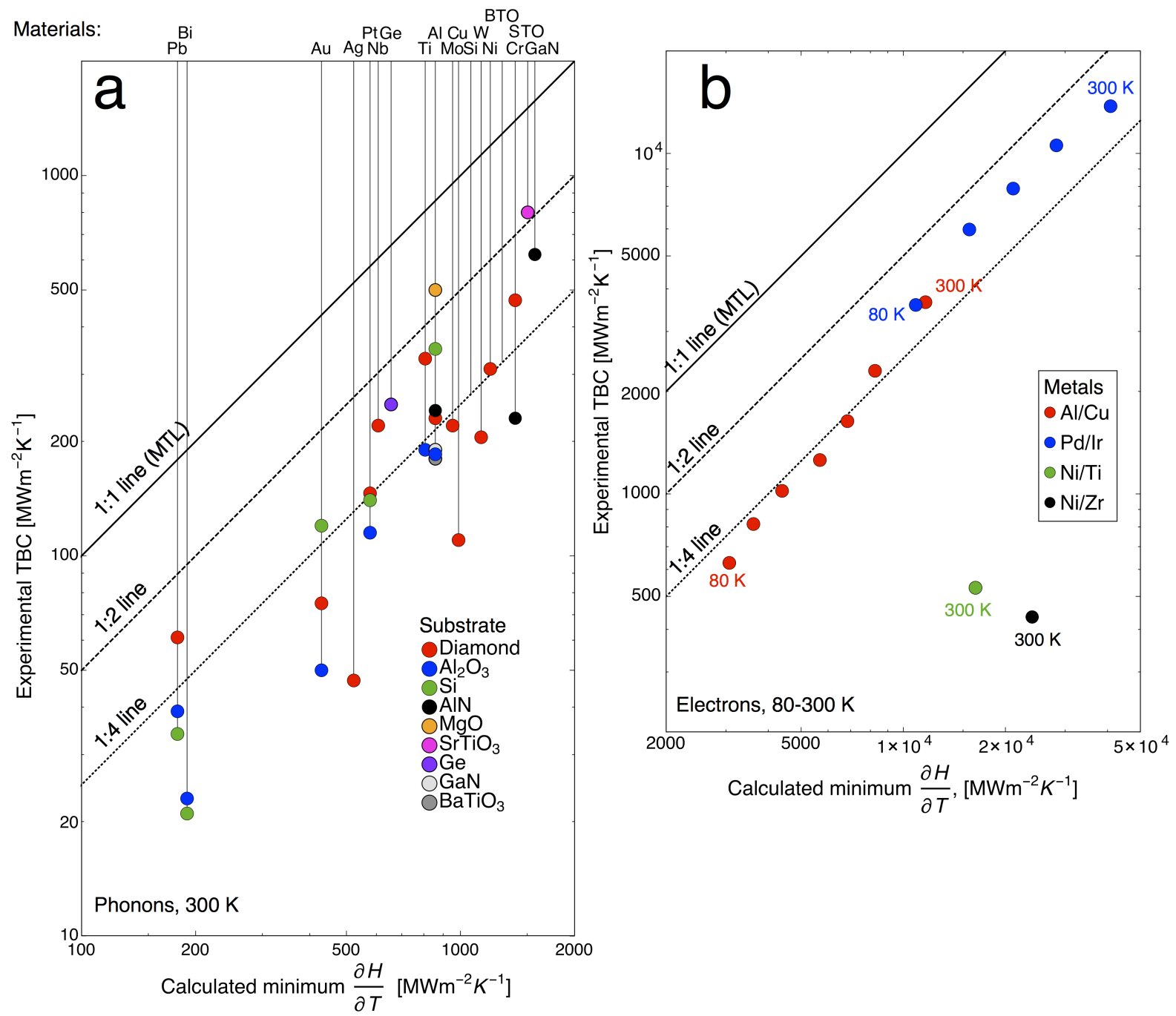

Figure 6 Collected experimental TBC values for systems dominated by $(a)$ phonons and $(b)$ electrons, organized to compare with the minimum irradiance framework (Equations 5 and 11). Each point in panel $a$ represents a unique materials pair: In analogy to Figure 5, the material with the weaker $\partial \mathrm{H} / \partial \mathrm{T}$ locates the point along the bottom axis (with the specific material indicated by the corresponding ticks along the top axis), whereas the material with the stronger $\partial \mathrm{H} / \partial \mathrm{T}$ is encoded by the point color. The $1: 1$ line corresponds to the maximum transmission limit (MTL), i.e., $\alpha_{\min \rightarrow \max }=1$, with the other two lines corresponding to $\alpha_{\min \rightarrow \max }$ of $\frac{1}{2}$ and $\frac{1}{4}$. Panel $b$ also includes temperature-dependent data below $300 \mathrm{~K}$. Phonon data are from References $\underline{2}, \underline{18}, \underline{36}-\underline{-38}$, $\underline{44}, \underline{46}, \underline{49}, \underline{53}, \underline{125}, \underline{128}, \underline{130}, \underline{131}, \underline{133}, \underline{136}$, and $\underline{137}$. Electron data are from References $\underline{1}, \underline{51}$, and $\underline{138)}$.

\subsection{Interfacial Bonding Strength and Stiffness}

The phonon transmission coefficients $\alpha$ depend critically on the atomic-scale details of the interfacial bonding and can vary widely in real interfaces (factors of 5 or 10 are not uncommon), depending on how exactly the surface was prepared. Indeed, as illustrated schematically in Figure 1a, the detailed structure of the interface can dramatically impact $\mathrm{TBC}$, be it through an interfacial layer [whether interdiffused $(\underline{16}, \underline{40}, \underline{133}, \underline{139})$ or deliberately added $(\underline{10}, \underline{140}-\underline{-142})]$, its roughness $(\underline{41}, \underline{143})$, or simply the substrate's chemical termination $(\underline{18}, \underline{126}, \underline{128})$. 
Using the diamond/metal system as an example, Figure 5 demonstrates the strong impact that the details of the interface formation process can have on the experimental TBC. TBC is significantly lower for interfaces on as-received diamonds. This reduction is caused by poor adhesion (132) due to organic residues (128). Similarly, films made by sputtering, a process that promotes adhesion (144), tend to have higher TBCs with clean diamond than do their evaporated counterparts (125). Finally, subjecting metal/diamond interfaces to hydrostatic pressures above 10--20 GPa [see the diamond anvil cell (DAC) points in Figure 5], thought to enable bond-enhancing chemical reactions, leads to the highest TBCs for any given system (130).

Electron-dominated-TBC results are also highly sensitive to interface quality. The much lower TBCs seen for the $\mathrm{Ni} / \mathrm{Zr}$ and $\mathrm{Ni} / \mathrm{Ti}$ couples in Figure $\boldsymbol{6} \boldsymbol{b}$ can be explained by the fact that those systems can react together to form intermetallic phases, thereby precluding the existence of abrupt, high-quality interfaces and thus decreasing $\langle\alpha\rangle$.

Figure 7 presents four experimental examples highlighting the strong impact of interfacial bonding on $h$ through $\alpha$. Figure 7a shows a compelling example using the TBC between a quartz substrate and a transfer-printed gold layer, which sandwich a self-assembled monolayer (SAM) of variable bonding strength (140). Increasing the density of covalent bonds between the SAM and the gold improves the TBC between gold and quartz by nearly a factor of 2, which also exhibits a physically satisfying correlation with improved adhesion as measured using a laser spallation method. Additionally, incorporating a Ti adhesion layer between Al and graphite approximately doubles the measured TBC (10). A similar enhancement has been observed with a $\mathrm{Ti}$ adhesion layer for the gold $/ \mathrm{SiO}_{2} \mathrm{TBC}(\underline{146})$. 


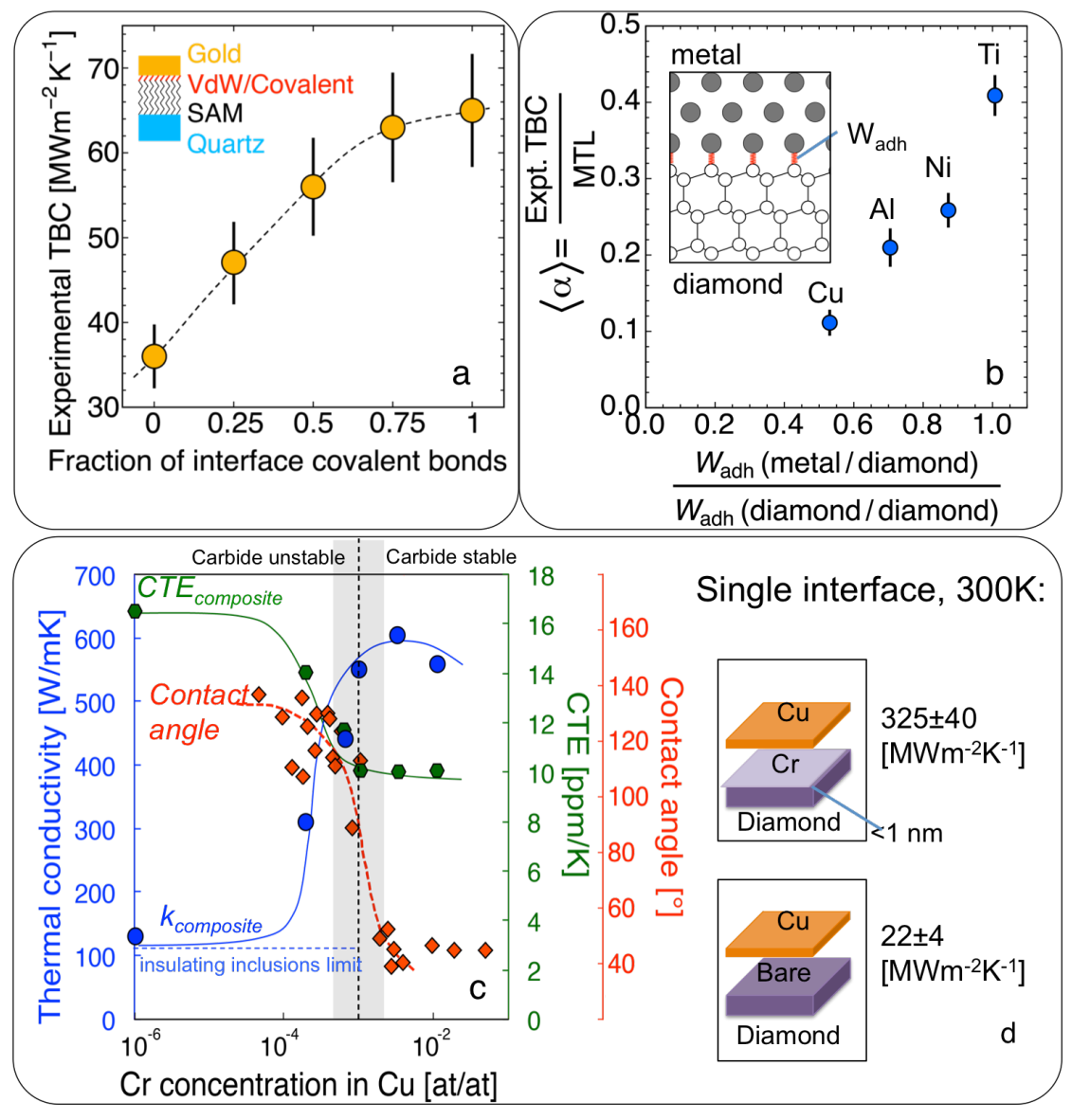

Figure 7 Representative experimental results showing the effects of adhesion on increasing $\langle\alpha\rangle$ and thus TBC. (a) Measurements by Losego et al. (140) show the impact of increasing the proportion of covalent versus van der Waals (vdW) bonds at a gold/quartz interface linked using self-assembled monolayers (SAMs). The much stronger covalent bonds increase TBC twofold. (b) The average transmission coefficient $\left\langle\alpha_{\min \rightarrow \max }\right\rangle$ increases monotonically with the strength of interfacial bonding, as represented by the work of adhesion, for a series of four metals on diamond. $\left\langle\alpha_{\min \rightarrow \max }\right\rangle$ is defined as the ratio of the measured TBC (44) to the calculated MTL (perfect-transmission) limit. Diamond's work of cohesion is $W_{\text {adh }}=5.61 \mathrm{~J} / \mathrm{m}^{2}$. (c) Effect of adding $\mathrm{Cr}$ to a $\mathrm{Cu}$ matrix in $\mathrm{Cu}$ /diamond composites formed by liquid-metal infiltration (16). For $\mathrm{Cr}$ concentrations above $\sim 10^{-3}$, a $\mathrm{Cr}$ carbide layer forms at the surface of the diamond particles. This interlayer dramatically increases the affinity between $\mathrm{Cu}$ and diamond, as manifested by three simultaneous property transitions: increased wettability [reduced contact angle; red data (145)], reduced coefficient of thermal expansion (CTE) (green data) from that of pure copper $(17 \mathrm{ppm} / \mathrm{K})$ to a weighted average with diamond $(1 \mathrm{ppm} / \mathrm{K})$, and a fivefold increase in $k_{\text {comp }}$ (blue data). Without the bridging carbide, $\mathrm{CTE}_{\text {comp }}$ and $k_{\text {comp }}$ behave as if the diamonds were not even present. (d) In a complementary single-interface study, a sub-1-nm layer of $\mathrm{Cr}$ was found to enhance the $\mathrm{TBC}$ between $\mathrm{Cu}$ and diamond 15-fold (unpublished TDTR measurements from Reference 131), consistent with the increase in $k_{\text {comp }}$ from panel $c$.

Figure $7 \boldsymbol{b}$ shows how the work of adhesion $\left(W_{\text {adh }}\right)$ of a metal/diamond interface can be linked to its TBC. This is clearly illustrated by normalizing each measured TBC to the corresponding MTL calculation $\left(h_{\mathrm{MTL}}=\left[\frac{\partial H}{\partial T}\right]_{\min }\right)$, thereby giving $\left\langle\alpha_{\min \rightarrow \max }\right\rangle$. The clear trend is $\left\langle\alpha_{\min \rightarrow \max }\right\rangle$ increasing with $W_{\text {adh }}$, which correlates with the interfacial bonding stiffness.

Similar effects of adhesion on TBC are well known for MMCs $(\underline{16}, \underline{121}, \underline{147}, \underline{148})$. In particular, because $\mathrm{Cu}$ and $\mathrm{Ag}$ bond weakly to diamond, incorporating a bond-strengthening 
metallic carbide at the interface has become quite standard. Figure $7 c$ provides an exemplary case study of this approach. Adding small quantities of $\mathrm{Cr}$ to the $\mathrm{Cu}$ matrix causes $\mathrm{Cr}$ carbide formation for concentrations above $\sim 10^{-3} \mathrm{Cr} / \mathrm{Cu}$. This carbide dramatically improves the adhesion between $\mathrm{Cu}$ and diamond, and this improvement is independently seen through decreased coefficient of thermal expansion (CTE) [diamond ( 1 ppm/K) having a much lower $\mathrm{CTE}$ than $\mathrm{Cu}(\sim 17 \mathrm{ppm} / \mathrm{K})(\underline{16})]$ and in the reduced $\mathrm{Cu} /$ diamond contact angle. This improved adhesion also dramatically increases $k_{\text {comp }}$ more than fivefold, which, through the use of Equation 16, can be understood as the $\mathrm{Cu} /$ diamond $\mathrm{TBC}$ increasing from $\sim 0.5$ to 70 $\mathrm{MW} /\left(\mathrm{m}^{2} \cdot \mathrm{K}\right)$ due to the bridging interfacial carbide. Figure $7 \boldsymbol{d}$ further confirms this striking interfacial effect by comparing TDTR measurements of two $\mathrm{Cu} /$ diamond samples, with one diamond as received and the other diamond terminated with an ultrathin $(<1-\mathrm{nm}) \mathrm{Cr}$ carbide layer showing 15-fold increase in TBC. Similarly large interfacial effects have also been observed in $\mathrm{Al} /$ diamond MMCs. For example, estimating the TBC by using $k_{\mathrm{comp}}$ data from Reference 147 suggests an $\mathrm{Al} /$ diamond TBC that varies $\sim 100$-fold as the interfacial adhesion is improved by changing the production process to promote stronger interfacial bonding $(\underline{121})$.

\subsection{Finite-Thickness Interfacial Interlayers}

Interfacial layers between matrix and inclusions in MMCs can be crucial for good bonding and efficient heat transfer between matrix and inclusions $(\underline{148}, \underline{149})$. However, their chemical nature, crystallinity, and thickness may affect TBC as well. No significant impact was found (beyond the added resistance of the layer itself) of an amorphous $\mathrm{Al}_{2} \mathrm{O}_{3}$ [5] or a carbide [9] interlayer between metals and diamond, suggesting that a reaction layer could be present even in nominally pristine interfaces and could influence the behavior of these interfaces. Molecular dynamics studies also suggest that adding an interlayer with acoustic properties that transition between two otherwise mismatched materials can improve the thermal transport between them $(\underline{150}, \underline{151})$. Conversely, increasing the thickness of a silicide interlayer from 10 to $15 \mathrm{~nm}$ in a $\mathrm{Cr} / \mathrm{Si}$ system was measured to degrade its $\mathrm{TBC}$ by $\sim 40 \%$ $(\underline{152})$.

\subsection{Substrate Anisotropy}

All crystalline solids exhibit anisotropy in their phonon dispersion relation, causing their $H$ to vary with direction. Such phonon-focusing effects are expected to influence TBC and would help explain (153) the very low TBCs $\left[\sim 10-100 \mathrm{MW} /\left(\mathrm{m}^{2} \cdot \mathrm{K}\right)\right]$ observed between metals and 
$c$-axis-oriented graphite $(\underline{10}, \underline{135})$. Figure 8 summarizes the limited available data that directly compare TBC on two different crystal faces with otherwise identically prepared interfaces. For Si (cubic symmetry), no significant difference between $<100>$-oriented substrates and $<111>$-oriented substrates was observed for three different metals. A modest orientation effect was observed in the $\mathrm{Al} /$ sapphire TBC, attributed to sapphire's anisotropic crystal structure. However, Figure 8 should not be interpreted as ruling out strong orientation effects even for these substrates because, for all the couples presented, the TBC-limiting irradiance $\left[\frac{\partial H}{\partial T}\right]_{\min }$ is actually from the metal side, so these data are only weakly sensitive to the directionality of $H$ from the substrate side. Lastly, the strongest apparent anisotropy effect in Figure 8 is for the Al/diamond system when the diamond is terminated with hydrogen (125). However, this effect is explained not by an $H$ anisotropy of the diamond, but by the [111] face having a higher surface density of hydrogen (which is a weak bond) than the [100] face.

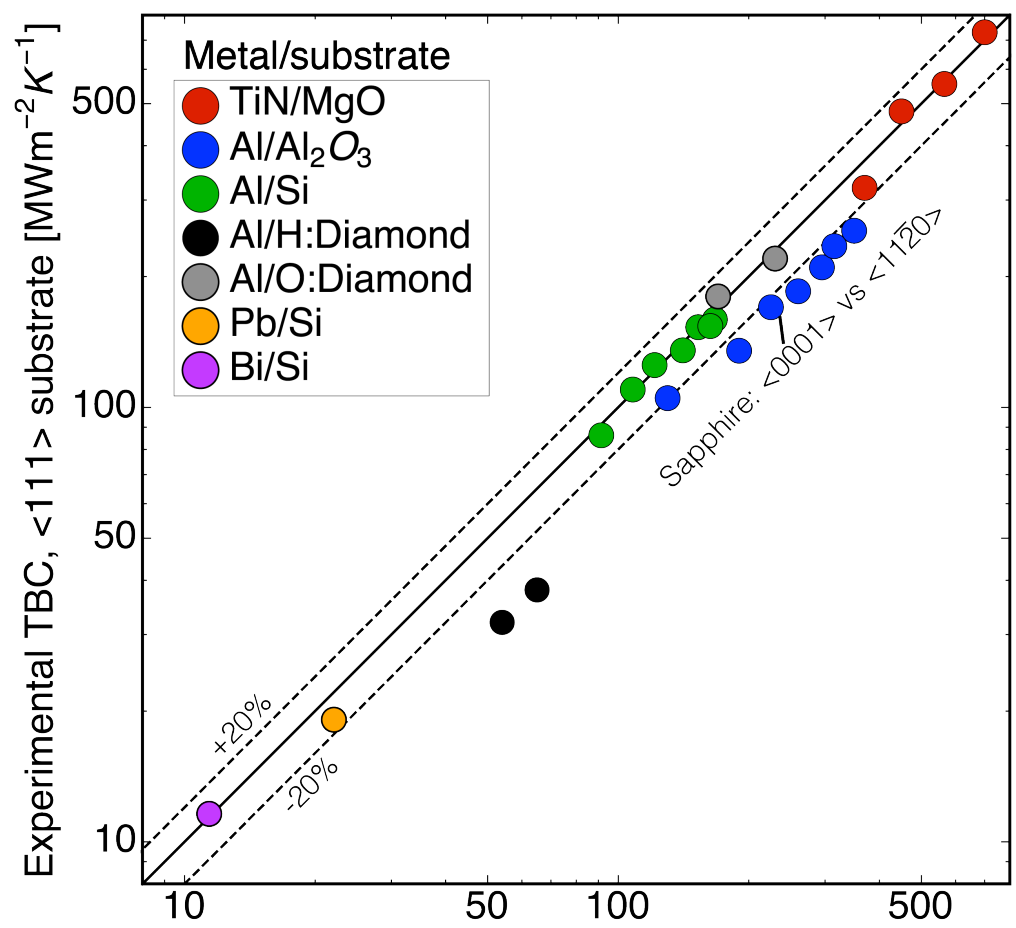

Experimental TBC, $<100>$ substrate $\left[\mathrm{MWm}^{-2} \mathrm{~K}^{-1}\right]$

Figure 8 Comparison of TBC on $<100>$-oriented substrates versus $<111>$-oriented substrates $(<0001>$ versus $<1120>$ for sapphire) as measured by TDTR around room temperature. Although there is little substrate orientation effect in this data set, for these couples the weak-irradiance side is the metal, so the measured TBC is expected to be relatively insensitive to any irradiance variations from the substrate side. The most prominent deviation from the 1:1 line is for $\mathrm{Al}$ on hydrogen-terminated diamond, but this deviation is actually because of known stronger adhesion on $\langle 100>$ faces (i.e., larger $\langle\alpha>$ ) for this system. Data from References $\underline{2}, \underline{53}, \underline{125}$, and 154. 


\subsection{Interfacial Roughness}

Intuitively, TBC could be influenced by roughness whose scale is comparable to or larger than the wavelengths of the thermal phonons (32), which is practically always the case for real interfaces at room temperature. However, the notion of direct roughness effects on TBC should be carefully treated for several reasons.

First, the most common metric, the rms roughness, is a single number. The surface-area enhancement and average slope should also play essential roles, but even the most basic quantification of the roughness spectrum is rarely reported. Second, the choice of roughness measurement tool has a significant influence on the reported roughness (155). Moreover, typical AFM tips have a radius of approximately $10 \mathrm{~nm}$ at best, thereby underestimating finerscale roughness (156). Finally, the processing method (e.g., etching) used to control the interfacial roughness may also change the surface chemistry, and disentangling the direct effects of roughness from the chemistry-induced changes in $\langle\alpha\rangle$ may not be possible.

Experimental evidence by Hopkins and colleagues (41, 143, 157) suggests that, for an $\mathrm{Al} / \mathrm{Si}$ interface, increasing interface roughness degrades the TBC. Measurements on the $\mathrm{Rh}: \mathrm{Fe} / \mathrm{Al}_{2} \mathrm{O}_{3}$ system by Swartz \& Pohl (26) also suggest a decrease in conductance with increasing interfacial roughness. Molecular dynamics calculations have supported both possibilities $(\underline{67}, \underline{158})$. Measurements by Edtmaier et al. (159) found that a roughening treatment applied to diamond particles improved $k_{\text {comp }}$ of an MMC made by liquid Al infiltration. However, the roughening treatment involved strong acids, which terminate the diamond surface with oxygen $(\underline{160})$ and may thus improve TBC due to this effect (recall Figure 5) rather than the roughness (106, 128).

A related geometric consideration which could potentially influence TBC is curvature of the interface, for example in the case of grain boundaries. Grain boundary curvature with high spatial frequencies can be considered as in the roughness discussion just presented. More gradual grain boundary curvature could conceivably impact the TBC through the angular dependence of $H$ due to phonon focusing effects, even in a cubic material, as discussed in Section 4.5. However, neither of these potential grain boundary curvature effects on TBC have been clearly documented in the experimental literature.

\subsection{Hydrostatic Pressure}

Hsieh et al. (39) and Hohensee et al. (130) showed that applying a hydrostatic (isotropic) pressure from 0 to $\sim 10 \mathrm{GPa}$ on clean $\mathrm{Al} / \mathrm{SiC}$ and $\mathrm{M} /$ diamond $(\mathrm{M}=\mathrm{Pt}, \mathrm{Au}, \mathrm{Pb}, \mathrm{Al})$ interfaces moderately increases the measured TBC (by tens of percent up to a factor of two), due to 
changes in the dispersion relations and thus $H$. Pressure effects on TBC are much more dramatic, up to eightfold, for purposely weakened interfaces (native $\mathrm{SiO}_{2}$ on $\mathrm{SiC}$, or incorporating a graphene sheet at the interface). At even higher pressures [up to $50 \mathrm{GPa}$ (130)], weak interfaces undergo a permanent change thought to improve the interfacial bonding and thus $\alpha$. The resulting TBCs are as high as or higher than any other experimental reports for the same couple (see the DAC points in Figure 5).

\subsection{Other Effects}

Confinement and dislocation density effects have also been briefly investigated. Krenzer et al. (161) used ultrathin Bi layers to explore possible effects of phononic confinement on TBC through $\langle\alpha\rangle$. Despite film thicknesses down to $2.5 \mathrm{~nm}$, the measured TBC remained constant. Calculations suggest that measurable phonon quantization effects would require film thicknesses close to a monolayer (at $T \sim 100 \mathrm{~K}$ and up), which is an experimental challenge and calls into question any model based on bond stiffnesses from bulk Bi.

The effects of dislocation density have also been investigated in the context of in-plane dislocation spacings as small as $20 \mathrm{~nm}$. Measurements of two GaSb epitaxial films (162) revealed a reduction in TBC in the film with increased dislocation density, although the same film also had a higher surface roughness, complicating the interpretation. A related dislocation density study of the $\mathrm{Bi} / \mathrm{Si}$ system (이) found no effect.

\section{CONCLUSION AND OUTLOOK}

\subsection{Key Physical Insights}

The guiding perspective of this review is that TBC is determined by two key phenomena (Figure 1): $(a)$ the irradiance of heat carriers bombarding an interface, $H_{i}$, and $(b)$ their corresponding transmission probabilities, $\left\langle\alpha_{i j}\right\rangle$. The irradiance depends on each material's heat capacity and carrier velocity through Equation 1 or Equation 7. The two materials are often highly mismatched, in which case TBC is limited by the material with the weaker $\frac{\partial H}{\partial T}$ (e.g., Figure 6). The average transmission probabilities $\langle\alpha\rangle$ are complex and remain hard to predict, calling into question the suitability of the classic AMM and DMM. The experiments collected above show that surface preparation and chemistry routinely impact $\langle\alpha\rangle$ by factors of 2 to 5-and, in some cases, by a factor of more than 100-for a given materials pair

(Figures 5 and 7). Nevertheless, the simple limit of $\alpha_{\min \rightarrow \max }=1$, that is, the MTL (Equation 
11), proves to be a useful upper bound, and most of the high-quality interfaces surveyed above exhibit TBCs within a factor of $\frac{1}{5}$ or even $\frac{1}{2}$ of the MTL.

\subsection{Major Advances over the Last 25 Years}

The major theoretical advances have been computational. Molecular and lattice dynamics methods have been refined and complemented by Green's function and wave packet methods. Atomistic Green's functions using DFT-derived potentials for both bulk and interfacial atoms are promising, although realistic quantitative calculations have yet to be made. Additionally, a growing body of experimental evidence is steering the theoretical understanding from an acoustic or diffuse mismatch picture back to a broader view based on heat carrier irradiance. The perfect-transmission approximation (the MTL) consistently overestimates measurements on high-quality systems by a factor of 2 to 5 but seems to better explain general trends in temperature or when different materials are compared.

On the experimental side, thermally modulated techniques, particularly the workhorse TDTR, have enabled a wealth of measurements. The greatest fundamental challenge now is preparing chemically clean, well-understood interfaces. TBC experimentalists have explored various materials science parameters such as substrate orientation and dispersion relation, interfacial layers and roughness, and hydrostatic pressure. Their collected results provide invaluable experimental grounding for thermal design at the nanoscale, with broad technological relevance from microelectronics to LEDs.

\subsection{Needs and Opportunities}

The TBC community appears close to realizing good agreement between experiments and computations without any free parameters. Achieving such predictive power across a range of systems would be a major milestone but requires further advances in both areas, especially at the interface:

- Computational: Moving beyond theoretically perfect systems is probably the greatest challenge. The interatomic potentials must be highly accurate to capture realistic bonding, defects, and anharmonicities throughout the interfacial region and to provide the deepest understanding of TBC. A theoretical framework to rigorously treat interfaces using first-principles approaches (beyond the one provided by atomistic Green's functions) has yet to be developed.

- Experimental: Identifying and producing clean, epitaxial systems in materials pairs with highly mismatched irradiances would give excellent grounds for comparison with 
computational results. More thorough, nonthermal characterization of interfaces as a complement to existing measurement techniques is needed to better understand TBC fundamentals at the nanoscale. Extremely fine-scale structural characterization is needed because, at ambient temperature and above, the dominant phonon wavelengths are of the order of one interatomic distance, so the interface should be well characterized at that same scale.

Among materials classes, amorphous materials and metal/metal interfaces will benefit from deeper investigations. Interfaces involving amorphous materials have not been carefully addressed by modeling or experiment, in spite of their growing significance in state-of-the-art microelectronics. Modeling their TBC may involve some subtleties because the vibrational energy transfer in amorphous materials is extremely short range $(163,164)$, challenging even the basic Landauer-Büttiker picture. Regarding electron-dominated interfaces, only two systems have been investigated in depth (Figure 6b), although neither was highly mismatched. Thus, more measurements using metal pairs with widely contrasting Fermi velocities and Sommerfeld parameters will enable a more compelling assessment of current theories.

From an applications perspective, work is needed to understand the impact of each fabrication step on the TBC of any given interface. We show above that TBC can be extremely sensitive to interfacial treatments using well-controlled chemistry in the lab (e.g., Figures 5 and 7), but much less work has been done for industrially relevant interfacial processing steps. For example, does stripping photoresist impact the TBC between two successive layers in a stack; would a plasma cleaning step improve it; and if so, why? Such research is challenging because it requires making interfaces "dirty" in a reproducible way, but the resulting understanding could enable major thermal management improvements at only modest cost.

\section{DISCLOSURE STATEMENT}

The authors are not aware of any affiliations, memberships, funding, or financial holdings that might be perceived as affecting the objectivity of this review.

\section{ACKNOWLEDGMENTS}

C.M. acknowledges funding from the Swiss National Science Foundation under grant P2ELP2-152177. C.D. acknowledges funding from the U. S. National Science Foundation 
under grants 0854554 and 1358370 . We thank Geoffrey P. Wehmeyer for his careful reading of part of the manuscript and Richard B. Wilson for helpful discussions.

\section{REFERENCES}

1. Wilson RB, Cahill DG. 2012. Experimental validation of the interfacial form of the Wiedemann-Franz law. Phys. Rev. Lett. 108:255901

2. Lyeo H-K, Cahill DG. 2006. Thermal conductance of interfaces between highly dissimilar materials. Phys. Rev. B 73(14):144301

3. Tas G, Stoner RJ, Maris HJ, Rubloff GW, Oehrlein GS, Halbout JM. 1992. Noninvasive picosecond ultrasonic detection of ultrathin interfacial layers: $\mathrm{CFx}$ at the $\mathrm{Al} / \mathrm{Si}$ interface. Appl. Phys. Lett. 61(6):1787--89

4. Rafailov EU, Cataluna MA, Sibbett W. 2007. Mode-locked quantum-dot lasers. Nat. Photonics 1(7):395--401

5. Challener WA, Peng C, Itagi AV, Karns D, Peng W, et al. 2009. Heat-assisted magnetic recording by a near-field transducer with efficient optical energy transfer. Nat. Photonics 3(April):220--24

6. Kemp A, Valentine G, Hopkins J-M, Hastie JE, Smith SA, et al. 2005. Thermal management in vertical-external-cavity surface-emitting lasers: analysis of a heatspreader approach. IEEE J. Quantum Electron. 41(2):148--55

7. Gačević Z, Rossbach G, Butté R, Réveret F, Glauser M, et al. 2013. Q-factor of $(\mathrm{In}, \mathrm{Ga}) \mathrm{N}$ containing III-nitride microcavity grown by multiple deposition techniques. $J$. Appl. Phys. 114(23):233102

8. Jin Y, Shao C, Kieffer J, Pipe KP, Shtein M. 2012. Origins of thermal boundary conductance of interfaces involving organic semiconductors. J. Appl. Phys. 112:093503

9. Pettes MT, Shi L. 2009. Thermal and structural characterizations of individual single-, double-, and multi-walled carbon nanotubes. Adv. Funct. Mater. 19:3918--25

10. Schmidt AJ, Collins KC, Minnich AJ, Chen G. 2010. Thermal conductance and phonon transmissivity of metal-graphite interfaces. J. Appl. Phys. 107:104907

11. Hopkins PE, Baraket M, Barnat EV, Beechem TE, Kearney SP, et al. 2012. Manipulating thermal conductance at metal-graphene contacts via chemical functionalization. Nano Lett. 12(2):590--95

12. Heath JR. 2009. Molecular electronics. Annu. Rev. Mater. Res. 39:1--23

13. Liu CW, Östling M, Hannon JB. 2014. New materials for post-Si computing. MRS Bull. 39(8):658--62 
14. Cho J, Bozorg-Grayeli E, Kodama T, Francis D, Ejeckam F, et al. 2013. Improved thermal interfaces of GaN-diamond composite substrates for hemt applications. IEEE Trans. Compon. Packag. Manuf. Technol. 3(1):79--85

15. Zweben C. 2007. Advances in high-performance thermal management materials - a review. J. Adv. Mater. 39(1):3--10

16. Weber L, Tavangar R. 2007. On the influence of active element content on the thermal conductivity and thermal expansion of $\mathrm{Cu}-\mathrm{X}(\mathrm{X}=\mathrm{Cr}, \mathrm{B})$ diamond composites. Scr. Mater. 57:988--91

17. Abyzov AM, Kidalov SV, Shakhov FM. 2012. High thermal conductivity composite of diamond particles with tungsten coating in a copper matrix for heat sink application. Appl. Therm. Eng. 48:72--80

18. Kida M, Weber L, Monachon C, Mortensen A. 2011. Thermal conductivity and interfacial conductance of AIN particle reinforced metal matrix composites. J. Appl. Phys. 109:064907

19. Gengler JJ, Shenogin SVS, Bultman JE, Roy AK, Voevodin AA, Muratore C. 2012. Limited thermal conductance of metal-carbon interfaces. J. Appl. Phys. 112:094904

20. Tavangar R, Molina J-M, Weber L. 2007. Assessing predictive schemes for thermal conductivity against diamond-reinforced silver matrix composites at intermediate phase contrast. Scr. Mater. 56(5):357--60

21. Kaur S, Raravikar N, Helms BA, Prasher RS, Ogletree DF. 2014. Enhanced thermal transport at covalently functionalized carbon nanotude array interfaces. Nat. Commun. $5: 3082$

22. Reifenberg J, Kencke DL, Goodson KE. 2008. The impact of thermal boundary resistance in phase-change memory devices. IEEE Electron Device Lett. 29(10):1112-14

23. Ji H, Sellan DP, Pettes MT, Kong X, Ji J, et al. 2013. Enhanced thermal conductivity of phase change materials with ultrathin-graphite foams for thermal energy storage. Energy Environ. Sci. 7:1185--92

24. Costescu RM, Cahill DG, Fabreguette FH, Sechrist ZA, George SM, et al. 2004. Ultralow thermal conductivity in $\mathrm{w} / \mathrm{Al}_{2} \mathrm{O}_{3}$ nanolaminates. Science 303(5810):989

25. Prasher RS. 2005. Thermal boundary resistance of nanocomposites. Int. J. Heat Mass Transf. 48(23--24):4942--52 
26. Swartz ET, Pohl RO. 1989. Thermal boundary resistance. Rev. Mod. Phys. 61(3):605-68

27. Cahill DG, Wayne FK, Goodson KE, Mahan GD, Majumdar AK, et al. 2003. Nanoscale thermal transport. Appl. Phys. Rev. 93(2):793--818

28. Cahill DG, Braun PV, Chen G, Clarke DR, Fan S, et al. 2014. Nanoscale thermal transport. II. 2003--2012. Appl. Phys. Rev. 1:011305

29. Hopkins PE. 2013. Thermal transport across solid interfaces with nanoscale imperfections: effects of roughness, disorder, dislocations, and bonding on thermal boundary conductance. ISRN Mech. Eng. 2013:682586

30. Chen G. 2005. Nanoscale Energy Transport and Conversion: A Parallel Treatment of Electrons, Molecules, Phonons, and Photons. Oxford, UK: Oxford Univ. Press

31. Chen Z, Wei Z, Chen Y, Dames C. 2013. Anisotropic model for the thermal boundary conductance. Phys. Rev. B 87:125426

32. Little WA. 1959. The transport of heat between dissimilar solids at low temperatures. Can. J. Phys. 37:334--49

33. Graff KF. 1975. Wave Motion in Elastic Solids. New York: Dover

34. Prasher RS, Phelan PE. 2001. A scattering-mediated acoustic mismatch model for the prediction of thermal boundary resistance. J. Heat Transf. 123:105--12

35. Prasher RS. 2009. Accoustic mismatch model for thermal contact resistance of van des Waals contacts. Appl. Phys. Lett. 94:041905

36. Stoner RJ, Maris HJ. 1993. Kapitza conductance and heat flow between solids at temperatures from 50 to 300 K. Phys. Rev. B 48(22):16373--87

37. Hopkins PE, Salaway RN, Stevens RJ, Norris PM. 2007. Temperature-dependent thermal boundary conductance at $\mathrm{Al} / \mathrm{Al}_{2} \mathrm{O}_{3}$ and $\mathrm{Pt} / \mathrm{Al}_{2} \mathrm{O}_{3}$ interfaces. Int. J. Thermophys. 28:947--57

38. Stevens RJ, Smith AN, Norris PM. 2005. Measurement of thermal boundary conductance of a series of metal-dielectric interfaces by the transient thermoreflectance technique. J. Heat Transf. 127:315--22

39. Hsieh W-P, Lyons AS, Pop E, Keblinski P, Cahill DG. 2011. Pressure tuning of the thermal conductance of weak interfaces. Phys. Rev. B 84:184107

40. Beechem TE, Graham S, Hopkins PE, Norris PM. 2007. Role of interface disorder on thermal boundary conductance using virtual crystal approach. Appl. Phys. Lett. 90:054104 
41. Hopkins PE, Duda JC, Petz CW, Floro JA. 2011. Controlling thermal conductance through quantum dot roughening at interfaces. Phys. Rev. B 84:035438

42. Hopkins PE, Duda JC, Norris PM. 2011. Anharmonic phonon interactions at interfaces and contributions to thermal boundary conductance. J. Heat Transf. 133:062401

43. Snyder NS. 1970. Heat transport through helium. II. Kapitza conductance. Cryogenics 10:89--95

44. Monachon C, Schusteritsch G, Kaxiras E, Weber L. 2014. Qualitative link between work of adhesion and thermal conductance of metal/diamond interfaces. J. Appl. Phys. 115(12):123509

45. Dames C, Chen G. 2004. Theoretical phonon thermal conductivity of Si/Ge superlattice nanowires. J. Appl. Phys. 95(2):682

46. Wilson RB, Apgar BA, Hsieh W-P, Martin LW, Cahill DG. 2015. Thermal conductance of strongly bonded metal-oxide interfaces. Phys. Rev. B 91:115414

47. Reddy P, Castelino K, Majumdar AK. 2005. Diffuse mismatch model of thermal boundary conductance using exact phonon dispersion. Appl. Phys. Lett. 87:211908

48. Duda JC, Beechem TE, Smoyer JL, Norris PM, Hopkins PE. 2010. Role of dispersion on phononic thermal boundary conductance. J. Appl. Phys. 108(7):073515

49. Minnich AJ, Johnson JA, Schmidt AJ, Esfarjani K, Dresselhaus MS, et al. 2011. Thermal conductivity spectroscopy technique to measure phonon mean free paths. Phys. Rev. Lett. 107(9):095901

50. Kittel C. 2005. Introduction to Solid State Physics. New York: John Wiley and Sons. 8 th ed.

51. Gundrum BC, Cahill DG, Averback RS. 2005. Thermal conductance of metal-metal interfaces. Phys. Rev. B 72:245426

52. Majumdar AK, Reddy P. 2004. Role of electron-phonon coupling in thermal conductance of metal-nonmetal interfaces. Appl. Phys. Lett. 84(23):4768--70

53. Costescu RM, Wall MD, Cahill DG. 2003. Thermal conductance of epitaxial interfaces. Phys. Rev. B 67:054302

54. Wilson RB, Feser JP, Hohensee GT, Cahill DG. 2013. Two-channel model for nonequilibrium thermal transport in pump-probe experiments. Phys. Rev. B 88(14): 144305

55. Mahan GD. 2009. Kapitza thermal resistance between a metal and a nonmetal. Phys. Rev. B 79:075408 
56. Chernatynskiy A, Phillpot SR. 2013. Phonon-mediated thermal transport: confronting theory and microscopic simulation with experiment. Curr. Opin. Solid State Mater. Sci. 17(1):1--9

57. Giannozzi P, Baroni S, Bonini N, Calandra M, Car R, et al. 2009. QUANTUM ESPRESSO: a modular and open-source software project for quantum simulations of materials. J. Phys. Condens. Matter 21(39):395502

58. Kresse G, Furthmüller J. 1996. Efficient iterative schemes for ab initio total-energy calculations using a plane-wave basis set. Phys. Rev. B 54(16):11169--86

59. Guo H, Qi Y, Li X. 2010. Adhesion at diamond/metal interfaces: a density functional theory study. J. Appl. Phys. 107:033722

60. Siegel D, Hector L, Adams J. 2002. Adhesion, atomic structure, and bonding at the $\mathrm{Al}(111) / \alpha-\mathrm{Al}_{2} \mathrm{O}_{3}(0001)$ interface: a first principles study. Phys. Rev. B 65(8):085415

61. Schelling PK, Phillpot SR, Keblinski P. 2002. Comparison of atomic-level simulation methods for computing thermal conductivity. Phys. Rev. B 65:144306

62. Landry ES, McGaughey AJH. 2009. Thermal boundary resistance predictions from molecular dynamics simulations and theoretical calculations. Phys. Rev. B 80(16): 165304

63. Chantrenne P, Barrat J-L. 2004. Finite size effect in determination of thermal conductivities: comparing molecular dynamics results with simple models. J. Heat Transf. 126(4):577--85

64. Rajabpour A, Volz S. 2010. Thermal boundary resistance from mode energy relaxation times: case study of argon-like crystals by molecular dynamics. J. Appl. Phys. 108(9):094324

65. Young DA, Maris HJ. 1989. Lattice-dynamical calculation of the kapitza resistance between fcc lattices. Phys. Rev. B 40(6):3685--93

66. Schelling PK, Phillpot SR, Keblinski P. 2004. Kapitza conductance and phonon scattering at grain boundaries by simulation. J. Appl. Phys. 95:6082--91

67. Zhou XW, Jones RE, Kimmer CJ, Duda JC, Hopkins PE. 2013. Relationship of thermal boundary conductance to structure from an analytical model plus molecular dynamics simulations. Phys. Rev. B 87:094303

68. Sadav S, Che Y, Huang Z, Chen L, Kumar S, Fisher TS. 2014. The atomistic green's function method for interfacial phonon transport. Annu. Rev. Heat Transf. 89--145 
69. Saltonstall CB, Polanco CA, Duda JC, Ghosh AW, Norris PM, Hopkins PE. 2013. Effect of interface adhesion and impurity mass on phonon transport at atomic junctions. J. Appl. Phys. 113:013516

70. Zhang L, Keblinski P, Wang J-S, Li B. 2011. Interfacial thermal transport in atomic junctions. Phys. Rev. B 83(6):064303

71. Mingo N. 2006. Anharmonic phonon flow through molecular-sized junctions. Phys. Rev. B 74(12):125402

72. Tian Z, Esfarjani K, Chen G. 2012. Enhancing phonon transmission across a Si/Ge interface by atomic roughness: first-principle study with the Green's function method. Phys. Rev. B 86:235304

73. Schmidt C, Umlauf E. 1976. Thermal boundary resistance at interfaces between sapphire and indium. J. Low Temp. Phys. 22(5):597--611

74. Dames C. 2013. Measuring the thermal conductivity of thin films : 3 omega and related electrothermal methods. Annu. Rev. Heat Transf. 16:7--49

75. Cahill DG. 1990. Thermal conductivity measurement from 30 to $750 \mathrm{~K}$ : the $3 \omega$ method. Rev. Sci. Instrum. 61(12):802--8

76. Cahill DG, Bullen A, Lee S-M. 2000. Interface thermal conductance and the thermal conductivity of multilayer thin films. High Temp. High Press. 32:135--42

77. Lee SS-M, Cahill DG. 1997. Influence of interface thermal conductance on the apparent thermal conductivity of thin films. Microscale Thermophys. Eng. 1(1):37--41

78. Cahill DG. 1997. Heat transport in dielectric thin films and at solid-solid interfaces. Microscale Thermophys. Eng. 1(2):85--109

79. Borca-Tasciuc T, Liu W, Liu J, Zeng T, Song DW, et al. 2000. Thermal conductivity of symmetrically strained Si/Ge superlattices. Superlattices Microstruct. 28(3):199--206

80. Chen Z, Jang W, Bao W, Lau CN, Dames C. 2009. Thermal contact resistance between graphene and silicon dioxide. Appl. Phys. Lett. 95(16):161910

81. Schmidt AJ. 2013. Pump-probe thermoreflectance. Annu. Rev. Heat Transf. 16:159--81

82. Collins KC, Maznev AA, Cuffe J, Nelson KA, Chen G. 2014. Examining thermal transport through a frequency-domain representation of time-domain thermoreflectance data. Rev. Sci. Instrum. 85(12):124903

83. Malen JA, Baheti K, Tong T, Zhao Y, Hudgings JA, Majumdar AK. 2011. Optical measurement of thermal conductivity using fiber aligned frequency domain thermoreflectance. J. Heat Transf. 133:081601 
84. Regner KT, Majumdar S, Malen JA. 2013. Instrumentation of broadband frequency domain thermoreflectance for measuring thermal conductivity accumulation functions. Rev. Sci. Instrum. 84(6):064901

85. Yang J, Maragliano C, Schmidt AJ. 2013. Thermal property microscopy with frequency domain thermoreflectance. Rev. Sci. Instrum. 84:1--9

86. Cahill DG. 2004. Analysis of heat flow in layered structures for time-domain thermoreflectance. Rev. Sci. Instrum. 75(12):5119--22

87. Schmidt AJ, Chen X, Chen G. 2008. Pulse accumulation, radial heat conduction, and anisotropic thermal conductivity in pump-probe transient thermoreflectance. Rev. Sci. Instrum. 79:114902

88. Siemens ME, Li Q, Yang R, Nelson KKA, Anderson EH, et al. 2010. Quasi-ballistic thermal transport from nanoscale interfaces observed using ultrafast coherent soft X-ray beams. Nat. Mater. Lett. 9(Suppl.):26--30

89. Highland M, Gundrum BC, Koh YK, Averback RS, Cahill DG, et al. 2007. Ballisticphonon heat conduction at the nanoscale as revealed by time-resolved X-ray diffraction and time-domain thermoreflectance. Phys. Rev. B 76:075337

90. Hanisch-Blicharski A, Krenzer B, Kalus A, Frigge T, Horn-von Hoegen M, et al. 2012. Heat transport through interfaces with and without dislocation arrays. J. Mater. Res. 27(21):1--6

91. Maxwell JC. 1891. A Treatise on Electricity and Magnetism, Vol. 1. Oxford, UK: Clarendon

92. Einstein A. 1906. Eine neue Bestimmung der Moleküldimensionen. Ann. Phys. 19:289-306

93. Hashin Z. 1968. Assessment of self consistent scheme approximation-conductivity of particulate composites. J. Compos. Mater. 2(3):284--300

94. Hale DK. 1976. The physical properties of composite materials. J. Mater. Sci. 11:2105-41

95. Hashin Z. 1983. Analysis of composite-materials - a survey. J. Appl. Mech. ASME 50(3):481--505

96. Torquato S. 2002. Random Heterogeneous Media, Vol. 16. New York: Springer. 701 pp.

97. Milton GW. 2002. The Theory of Composites. Cambridge, UK: Cambridge Univ. Press. 719 pp. 
98. Miloh T, Benveniste Y. 1988. A generalized self-consistent method for the effective conductivity of composites with ellispoidal inclusions and cracked bodies. J. Appl. Phys. 63(3):789---96

99. Benveniste Y. 1987. Effective thermal conductivity of composites with a thermal contact resistance between the constituents: nondilute case. J. Appl. Phys. 61(8):2840-43

100. Hasselman PH, Johnson LF. 1987. Effective thermal conductivity of composites with interfacial thermal barrier resistance. J. Compos. Mater. 21:508--15

101. Regner KT, Freedman JP, Malen JA. 2015. Advances in studying phonon mean-freepath-dependent contributions to thermal conductivity. Nanoscale Microscale Thermophys. Eng. 19(3):183--205

102. Nan CW, Li XP, Birringer R. 2000. Inverse problem for composites with imperfect interface: determination of interfacial thermal resistance, thermal conductivity of constituents, and microstructural parameters. J. Am. Ceram. Soc. 83(4):848--54

103. Hasselman DPH, Donaldson KY, Thomas JR. 1993. Effective thermal-conductivity of uniaxial composite with cylindrically orthotropic carbon-fibers and interfacial thermal barrier. J. Compos. Mater. 27(6):637--44

104. Geiger AL, Hasselman PH, Donaldson KY. 1993. Effect of reinforcement particle size on the thermal conductivity of a particulate silicon carbide-reinforced aluminum-matrix composite. J. Mater. Sci. Lett. 12:420--23

105. Davis L, Artz B. 1995. Thermal conductivity of metal-matrix composites. J. Appl. Phys. 77(10):4954--60

106. Edtmaier C, Bauer E, Weber L, Tako ZS, Segl J, Friedbacher G. 2015. Temperature dependence of the thermal boundary conductance in $\mathrm{Ag}-3 \mathrm{Si} /$ diamond composites. Diam. Relat. Mater. 57:37--42

107. Edtmaier C, Bauer E, Tako ZS, Segl J. 2015. Thermal conductivity behaviour of $\mathrm{Al} /$ diamond and $\mathrm{Ag} /$ diamond composites in the temperature range $4 \mathrm{~K}<t<293 \mathrm{~K}$. Mater. Sci. Forum 825--826:197--204

108. Molina JM, Prieto R, Narciso J, Louis E. 2009. The effect of porosity on the thermal conductivity of Al-12 wt.\% Si/SiC composites. Scr. Mater. 60:582--85

109. Weber L, Sinicco G, Molina JM. 2010. Influence of processing route on electrical and thermal conductivity of $\mathrm{Al} / \mathrm{SiC}$ composites with bimodal particle distribution. J. Mater. Sci. 45(8):2203--9 
110. Every AG, Tzou Y, Hasselman PH, Raj R. 1992. The effect of particle size on the thermal conductivity of $\mathrm{ZnS} /$ diamond composites. Acta Metall. Mater. 40(1):123--29

111. Wang J, Yi XS. 2004. Effects of interfacial thermal barrier resistance and particle shape and size on the thermal conductivity of AlN/PI composites. Compos. Sci. Technol. $64: 1623--28$

112. Benveniste Y, Miloh T. 1999. Neutral inhomogeneities in conduction phenomena. $J$. Mech. Phys. Solids 47(9):1873---92

113. Geiger AL, Hasselmann DPH, Welch P. 1997. Electrical and thermal conductivity of discontinuously reinforced aluminum composites at sub-ambient temperature. Acta Mater. 45(9):3911--14

114. Hasselmann DPH, Johnson LF. 1987. Effective thermal conductivity of composites with interfacial thermal barrier resistance. J. Compos. Mater. 21:508--15

115. Weber L, Dorn J, Mortensen A. 2003. On the electrical conductivity of metal matrix composites containing high volume fractions of non-conducting inclusions. Acta Mater. 51:3199--212

116. Boehm HJ, Nogales S. 2008. Mori-Tanaka models for the thermal conductivity of composites with interfacial resistance and particle size distributions. Compos. Sci. Technol. 68(5):1181--87

117. Yamamoto Y, Imai T, Tanabe K, Tsuno T, Kumazawa Y, Fujimori N. 1997. The measurement of thermal properties of diamond. Diam. Relat. Mater. 6:1057--61

118. Novikov NV, Podoba AP, Perevertailo VM, Shmegera S V, Witek A. 2000. Effect of isotope content on the cubic boron nitride lattice thermal conductivity. Diam. Relat. Mater. 9:629--31

119. Abyzov AM, Kidalov SV, Shakhov FM. 2012. Filler-matrix thermal boundary resistance of diamond-copper composite with high thermal conductivity. Phys. Solid State 54(1):210--15

120. Kang Q, He X, Ren S, Zhang L, Wu M, et al. 2013. Preparation of copper-diamond composites with chromium carbide coatings on diamond particles for heat sink applications. Appl. Therm. Eng. 60:423--29

121. Monje IE, Louis E, Molina J-M. 2013. Optimizing thermal conductivity in gas-pressure infiltrated aluminum/diamond composites by precise processing control. Composites $A$ 48:9--14 
122. Hasselmann DPH, Donaldson KY, Liu J, Gauckler LJ, Ownby PD. 1994. Thermal conductivity of a particulate-diamond-reinforced cordierite matrix composite. J. Am. Ceram. Soc. 77(7):1757--60

123. Hasselmann DPH, Donaldson KY, Geiger AL. 1992. Effect of reinforcement particlesize on the thermal conductivity of a particulate silicon carbide-reinforced aluminum matrix composite. J. Am. Ceram. Soc. 75:3137--40

124. Monachon C, Hojeij M, Weber L. 2011. Influence of sample processing parameters on thermal boundary conductance value in an Al/AlN system. Appl. Phys. Lett. 98(9):091905

125. Monachon C, Weber L. 2013. Effect of diamond surface orientation on the thermal boundary conductance between diamond and aluminum. Diam. Relat. Mater. 39:8--13

126. Collins KC, Chen S, Chen G. 2010. Effects of surface chermistry on thermal conductance at aluminum-diamond interfaces. Appl. Phys. Lett. 97:083102

127. Moffat RJ. 1988. Describing the uncertainties in experimental results. Exp. Therm. Fluid Sci. 1(1):3--17

128. Monachon C, Weber L. 2013. Influence of diamond surface termination on thermal boundary conductance between Al and diamond. J. Appl. Phys. 113:183504

130. Hohensee GT, Wilson RB, Cahill DG. 2015. Thermal conductance of metal-diamond interfaces at high pressure. Nat. Commun. 6:6578

131. Monachon C. 2013. Thermal Boundary Conductance Between Metals and Dielectrics., $\mathrm{PhD}$ Thesis

132. Monachon C, Weber L. 2012. Thermal boundary conductance of transition metals on diamond. Emerg. Mater. Res. 1(2):89--98

133. Monachon C, Weber L. 2014. Thermal boundary conductance between refractory metal carbides and diamond. Acta Mater. 73:337--46

134. Gengler JJ, Sukesh R, Jones JG, Gord JR. 2012. Two-color thermoreflectance of various metal transducers with an optical parametric oscillator. Meas. Sci. Technol. 23:055205

135. Norris PM, Smoyer JL, Duda JC, Hopkins PE. 2012. Prediction and measurement of thermal transport across interfaces betwen isotropic solids and graphitic materials. $J$. Heat Transf. 134:02910

136. Oh D-W, Kim S, Rogers JA, Cahill DG, Sinha S. 2011. Interfacial thermal conductance of transfer-printed metal films. Adv. Mater. 23:5028--33 
137. Koh YK, Cao Y, Cahill DG, Jena D. 2009. Heat-transport mechanisms in superlattices. Adv. Funct. Mater. 19(4):610--15

138. Clemens BM, Eesley GL, Paddock CA. 1988. Time-resolved thermal transport in compositionally modulated metal films. Phys. Rev. B 37(3):1085--96

139. Schubert T, Ciupiński Ł, Zieliński W, Michalski A, Weißgärber T, et al. 2008. Interfacial characterization of $\mathrm{Cu}$ /diamond composites prepared by powder metallurgy for heat sink applications. Scr. Mater. 58(4):263--66

140. Losego MD, Grady ME, Sottos NR, Cahill DG, Braun PV. 2012. Effects of chemical bonding on heat transport across interfaces. Nat. Mater. Lett. 11:502--5

141. Monachon C, Weber L. 2014. Influence of a nanometric $\mathrm{Al}_{2} \mathrm{O}_{3}$ interlayer on the thermal conductance of an Al/(Si, diamond) interface. Adv. Eng. Mater. 15(9999):1--8

142. Zhou XW, Jones RE, Duda JC, Hopkins PE. 2013. Molecular dynamics studies of material property effects on thermal boundary conductance. Phys. Chem. Chem. Phys. 15(26):11078--87

143. Hopkins PE, Phinney LM, Serrano JR, Beechem TE. 2010. Effects of surface roughness and oxide layer on the thermal boundary conductance at aluminum/silicon interfaces. Phys. Rev. B 82:085307

144. Ohring M. 2002. Materials Science of Thin Films. San Diego, CA: Academic

145. Scott PM, Nicholas M, Dewar B. 1975. The wetting and bonding of diamonds by copper-base binary alloys. J. Mater. Sci. 10:1833--40

146. Duda JC, Yang C-YP, Foley BM, Cheaito R, Medlin DL, et al. 2013. Influence of interfacial properties on thermal transport at gold:silicon contacts. Appl. Phys. Lett. 102:081902

147. Ruch PW, Beffort O, Kleiner S, Weber L, Uggowitzer PJJ. 2006. Selective interfacial bonding in $\mathrm{Al}(\mathrm{Si})$-diamond composites and its effect on thermal conductivity. Compos. Sci. Technol. 66(15):2677--85

148. Abyzov AM, Kidalov SV, Shakhov FM. 2011. High thermal conductivity composites consisting of diamond filler with tungsten coating and copper (silver) matrix. J. Mater. Sci. 46(5):1424--38

149. Schubert T, Trinidade B, Weissgärber T, Kieback B. 2008. Interfacial design of Cubased composites prepared by powder metallurgy for heat sink applications. Mater. Sci. Eng. A 475:39--44 
150. Liang Z, Tsai H-L. 2011. Effect of thin film confined between two dissimilar solids on interfacial thermal resistance. J. Phys. Condens. Matter. 23:495303

151. English TS, Duda JC, Smoyer JL, Jordan DA, Norris PM, Zhigilei LV. 2012. Enhancing and tuning thermal phonon transport at vibrationally mismatched solid-solid interfaces. Phys. Rev. B 85:035438

152. Hopkins PE, Norris PM. 2006. Thermal boundary conductance response to a change in Cr/Si interfacial properties. Appl. Phys. Lett. 89:131909

153. Chen Z, Wei Z, Chen Y, Dames C. 2013. Anisotropic Debye model for the thermal boundary conductance. Phys. Rev. B 87(12):125426

154. Hopkins PE, Beechem TE, Duda JC, Hattar K, Ihlefeld JF, et al. 2011. Influence of anisotropy on thermal boundary conductance at solid interfaces. Phys. Rev. B $84: 125408$

155. Poon CY, Bhushan B. 1995. Comparison of surface roughness measurements by stylus profiler, AFM and non-contact optical profiler. Wear 190:76--80

156. Sedin DL, Rowlen KL. 2001. Influence of tip size on afm roughness measurements. Appl. Surf. Sci. 182(1--2):40--48

157. Duda JC, Hopkins PE. 2012. Systematically controlling Kapitza conductance via chemical etching. Appl. Phys. Lett. 100:111602

158. Choi WWI, Kim K, Narumanchi S. 2012. Thermal conductance at atomically clean and disordered silicon/aluminum interfaces: a molecular dynamics simulation study. J. Appl. Phys. 112:054305

159. Edtmaier C, Weber L, Tavangar R. 2009. Surface modification of diamonds in diamond/Al-matrix composite. Adv. Mater. Res. 59:125--30

160. Kawarada H. 1996. Hydrogen-terminated diamond surfaces and interfaces. Surf. Sci. Rep. 26:205--59

161. Krenzer B, Hanisch-Blicharski A, Schneider P, Payer T, Möllenbeck S, et al. 2009. Phonon confinement effects in ultrathin epitaxial bismuth films on silicon studied by time-resolved electron diffraction. Phys. Rev. B 80:024307

162. Hopkins PE, Duda JC, Clark SP, Hains CP, Rotter TJ, et al. 2011. Effect of dislocation density on boundary conductance across GaSb/GaAs interfaces. Appl. Phys. Lett. 98:161913

163. Cahill DG, Pohl RO. 1988. Lattice vibrations and heat transport in crystals and glasses. Annu. Rev. Phys. Chem. 39:93--121 
164. Allen PB, Feldman JL, Fabian J, Wooten F. 1999. Diffusons, locons and propagons: character of atomic vibrations in amorphous Si. Philos. Mag. B 79(11--12):1715--31 